The Free Internet Journal

for Organic Chemistry
Review

Arkivoc 2018, part ii, 252-279

Organic Chemistry

\title{
When nucleoside chemistry met hypervalent iodine reagents
}

Mahesh K. Lakshman and Barbara Zajc

Department of Chemistry \& Biochemistry, The City College of New York, 160 Convent Avenue, New York, NY 10031, USA, and The Ph.D. Program in Chemistry, The Graduate Center of the City University of New York, New York, NY 10016, USA

Email: mlakshman@ccny.cuny.edu, bzajc@ccny.cuny.edu

\section{Dedicated to Professor Kenneth Laali on the occasion of his $65^{\text {th }}$ birthday}

Received 07-28-2017

Accepted 10-22-2017

Published on line 12-21-2017

\section{Abstract}

There has been increasing use of hypervalent iodine reagents in the field of nucleoside chemistry. Applications span: (a) synthesis of nucleoside analogues with sulfur and seleno sugar surrogates, (b) synthesis of unusual carbocyclic and ether ring-containing nucleosides, (c) introduction of sulfur and selenium into pyrimidine bases of nucleosides and analogues, (d) synthesis of isoxazole and isoxazoline ring-containing nucleoside analogues, (e) involvement of purine ring nitrogen atoms for remote $\mathrm{C}-\mathrm{H}$ bond oxidation, and ( $\mathrm{f}$ ) metal-catalyzed and uncatalyzed synthesis of benzimidazolyl purine nucleoside analogues by intramolecular $\mathrm{C}-\mathrm{N}$ bond formation. This review offers a perspective on developments involving the use of hypervalent iodine reagents in the field of nucleoside chemistry that have appeared in the literature in the 2003-2017 time frame.

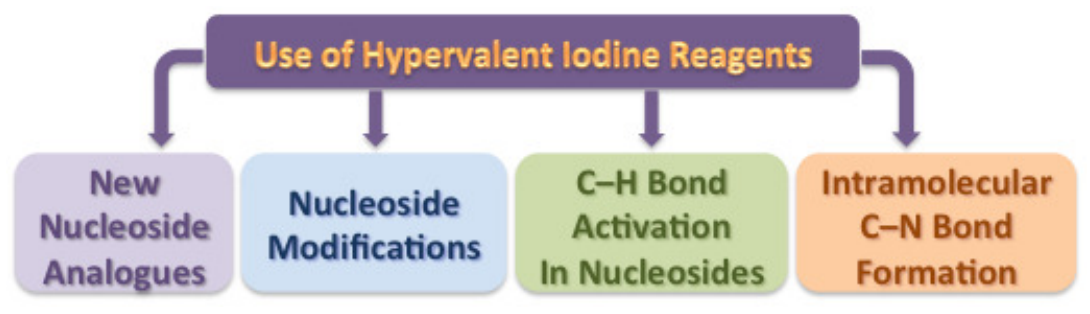

Keywords: Hypervalent iodine, nucleosides, $\mathrm{C}-\mathrm{N}$ bond formation, $\mathrm{C}-\mathrm{H}$ bond activation, nucleoside analogs 


\section{Table of Contents}

1. Introduction

2. Glycosylation-Type Reactions Leading to Nucleoside Analogues

3. Miscellaneous Hypervalent lodine Reagent Mediated Nucleoside Modifications

4. Purinyl N-Directed $\mathrm{C}-\mathrm{H}$ Bond Oxidation by PIDA

5. Cu-Catalyzed Intramolecular $\mathrm{C}-\mathrm{N}$ Bond Formation in Purine Nucleosides Using PIDA

6. Metal-Free Intramolecular C-N Bond Formation in Purine Nucleosides Using PIDA

7. Pd-Catalyzed and Metal-Free Intramolecular $\mathrm{C}-\mathrm{N}$ Bond Formation Leading to Isomeric Carbazolyl and Benzimidazolyl Purine Nucleoside Analogues Using PIDA

8. Conclusions

References

\section{Introduction}

Nucleosides are important biomolecules, present in all living systems and, thus, constitute a highly important class of compounds. They are fundamental building blocks of genetic material and because of this they are important scaffolds for development of new compounds with diverse applications. Predominantly, nucleosides and their analogues have been at the forefront in pharmaceuticals and medicine, as antiviral, anticancer, and antibiotic agents. ${ }^{1-10}$ In addition, they serve as biological probes and imaging agents. ${ }^{11-19}$

The eight natural nucleosides are shown in Figure 1. Two possess a purine heterocyclic aglycone and three a pyrimidine, and they all share commonalities in the saccharide moiety. The deoxyribosides possess an $\mathrm{H}$ atom at position 2' ( $X$ in Figure 1 ) whereas the ribosides have an $\mathrm{OH}$ at this position. In the quest for novel nucleoside analogues for the aforementioned applications, both the aglycone and the saccharide have been the subject of diverse modifications.

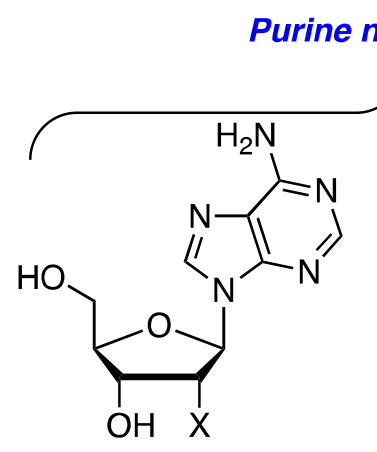

Deoxyadenosine: $\mathrm{X}=\mathrm{H}$ Adenosine: $\mathrm{X}=\mathrm{OH}$

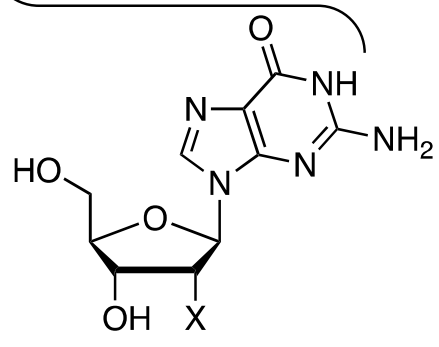

Deoxyguanosine: $\mathrm{X}=\mathrm{H}$ Guanosine: $\mathrm{X}=\mathrm{OH}$

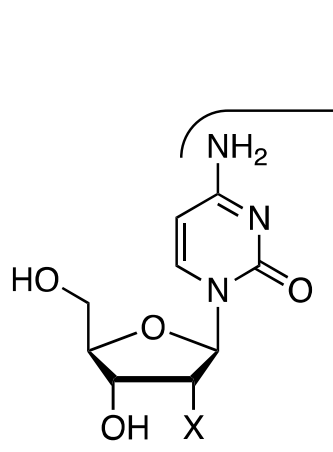

Deoxycytidine: $\mathrm{X}=\mathrm{H}$ Cytidine: $\mathrm{X}=\mathrm{OH}$
Pyrimidine nucleosides

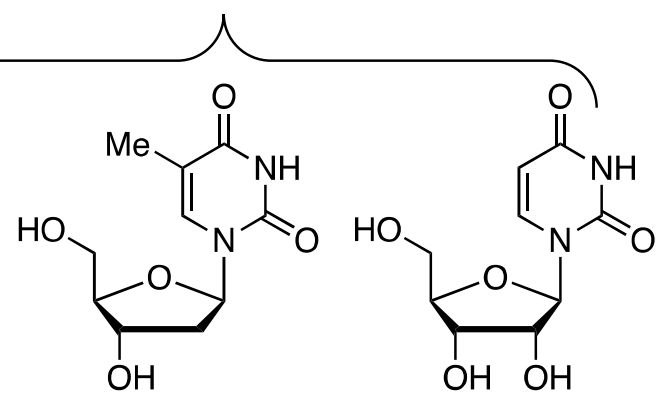

Thymidine

Uridine

Figure 1. The eight naturally occurring nucleosides.

Hypervalent iodine reagents are generally readily available and easy-to-handle. For this reason they are seeing wide applications in organic synthesis and methodology development. The most commonly encountered reagents have either $\lambda^{3}$ - or $\lambda^{5}$-iodine, and structures of the routinely encountered ones are shown in Figure 2 (PIDA = (diacetoxyiodo)benzene, PIFA = [bis(trifluoroacetoxy)iodo]benzene, IOB = 
iodosylbenzene or iodosobenzene, HTIB $=[($ hydroxy)(tosyloxy)iodo]benzene or Koser's reagent, IBX = 2iodoxybenzoic acid, and DMP = Dess-Martin periodinane).

$\lambda^{3}$ Reagents:<smiles></smiles>

PIDA

\section{$\lambda^{5}$ Reagents:}<smiles>O=C1OI(=O)(O)c2ccccc21</smiles><smiles>CC(C)(C)OC(=O)c1ccccc1</smiles>

PIFA<smiles>CC(=O)O[Te]1(OC(C)=O)(OC(C)=O)OC(=O)c2ccccc21</smiles><smiles>O=Ic1ccccc1</smiles>

$\mathrm{IOB}$<smiles>OI(O)c1ccccc1</smiles>

HTIB

Figure 2. Commonly encountered $\lambda^{3}$ and $\lambda^{5}$ hypervalent iodine reagents.

In this review, we describe some of the applications of hypervalent iodine reagents to the field of nucleoside modification.

\section{Glycosylation-Type Reactions Leading to Nucleoside Analogues}

In 2003, the use of hypervalent iodine reagents was demonstrated for the synthesis of pyrimidine nucleoside analogues containing a sulfur atom in the sugar-like portion. ${ }^{20}$ Initial experiments involved reactions of tetrahydrothiophene and thymine. With 2 equiv. of thymine and 6 equiv. each of $\mathrm{TMSOTf}$ and $\mathrm{Et}_{3} \mathrm{~N}$, and 1.2 equiv. of PIFA, in $\mathrm{CH}_{2} \mathrm{Cl}_{2}$ at $0{ }^{\circ} \mathrm{C}$, an excellent $80 \%$ yield of 1-(tetrahydrothiophen-2-yl)thymine was obtained.

The next step was the assessment of the reaction with tetrahydrothiophene derivatives decorated with additional hydroxyl groups. As shown in Scheme 1, cis-2-butene-1,4-diol 1 was converted to the protected dihydroxytetrahydrothiophene 2 via 8 steps ( $\mathrm{PMBz}=p$-methoxybenzoyl). Two hypervalent iodine reagents, PIFA and IOB, were tested for the "glycosylation". An anomeric mixture of compound 3 was obtained. Variation of conditions using PIFA altered the anomer ratio and the best $5: 1 \beta / \alpha$ ratio was obtained with 3 equiv. each of thymine, TMSOTf, and $2 \times 4$ equiv. of $\mathrm{Et}_{3} \mathrm{~N}$ (yield of 3 was $65 \%$ ). With IOB, only the $\beta$ anomer was formed (61\%).

Next a synthesis of a ribosyl analogue of uracil was sought. Starting from D-ribose, the tetrahydrothiophene 4 was synthesized. Evaluation of PIFA and IOB indicated the latter to be superior at $0{ }^{\circ} \mathrm{C}$. Whereas PIFA gave a $5: 1$ ratio of anomers, IOB gave exclusively the $\beta$-anomer 5 in $53 \%$ yield. The latter outcome is consistent with the formation of a thionium cation that is also stabilized by the neighboring PMBz ester. Final deprotection of the silyl and ester groups yielded the desired compound 6 (66\%). 


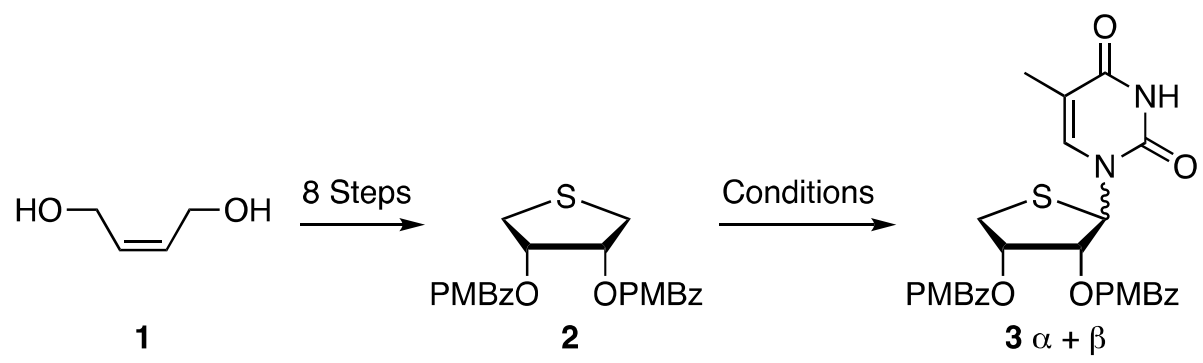

Conditions A: thymine, TMSOTf, $\mathrm{Et}_{3} \mathrm{~N}, \mathrm{PIFA}, \mathrm{CH}_{2} \mathrm{Cl}_{2}, 0^{\circ} \mathrm{C}(65 \%, \beta: \alpha 5: 1)$

Conditons $\mathrm{B}$ : thymine, TMSOTf, $\mathrm{Et}_{3} \mathrm{~N}, \mathrm{IOB}, \mathrm{CH}_{2} \mathrm{Cl}_{2}, 0^{\circ} \mathrm{C}(61 \%, \beta$ only $)$

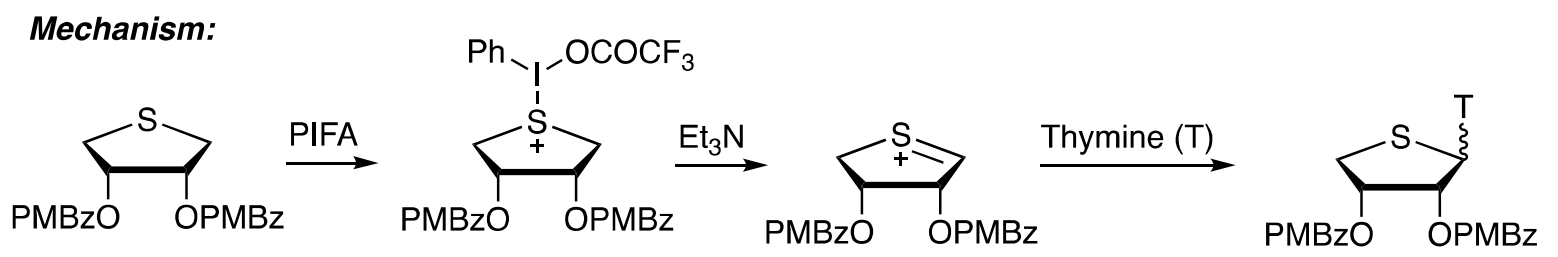

Scheme 1. Synthesis of a higher order thymine nucleoside analogue and a plausible mechanism.

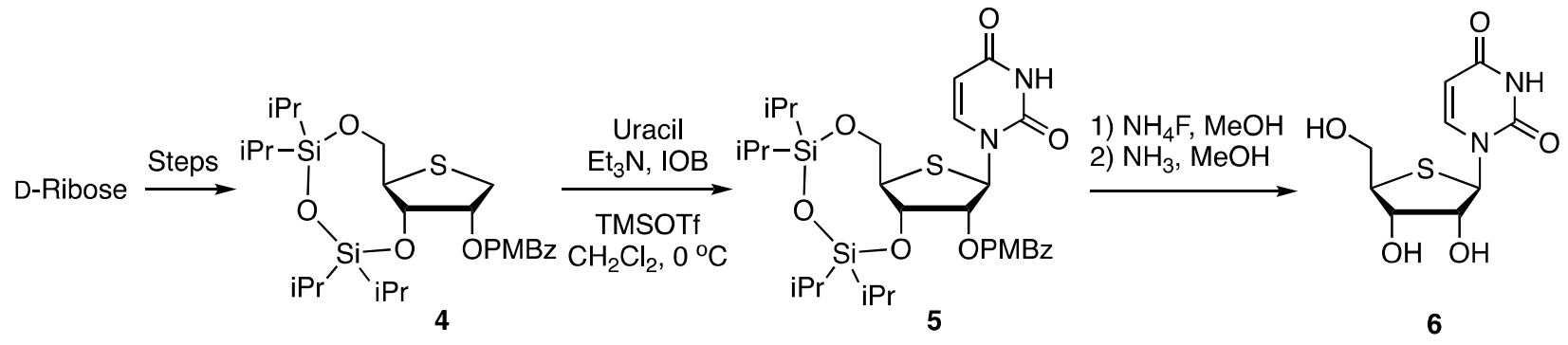

Scheme 2. Synthesis of a thio analogue of uridine.

In 2008, the synthesis of purine nucleoside analogues containing a thiosaccharide analogue was reported. ${ }^{21}$ As in Scheme 1, the dibenzoate analogue of tetrahydrothiophene diol 7 was utilized in initial reactions with three purine derivatives as shown in Scheme 3.

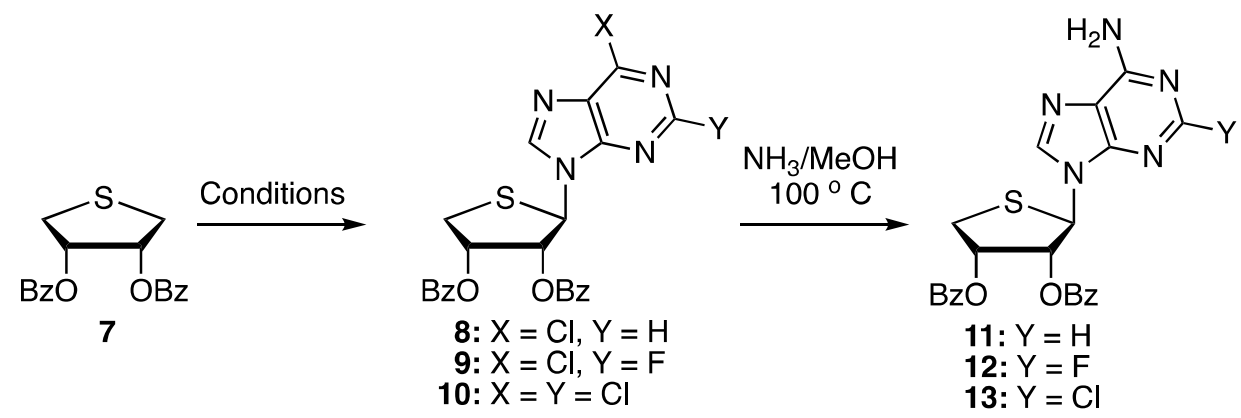

Scheme 3. Reactions of three purines with a tetrahydrothiophenediol dibenzoate.

Reaction of 6-chloropurine did not proceed with IOB, but product 8 (35\%) was obtained with 1.5 equiv. of PIFA, 10 equiv. each of TMSOTf and $\mathrm{Et}_{3} \mathrm{~N}$. Under these conditions, 2-fluoro-6-chloropurine and 2,6dichloropurine also reacted giving the respective products, 9 and 10, in 36 and $72 \%$ yields, respectively. In each case $\alpha / \beta$ anomers were observed; $1: 5$ with 6 -chloropurine, $1: 3$ with 2 -fluoro-6-chloropurine, and $1: 15$ 
with 2,6-dichloropurine. The isolated $\beta$-isomers were ammonolyzed to yield the C6-amino products (11: quantitative, 12: $81 \%$, and 13: $67 \%$ ).

Next four precursors, 15-18, were prepared from D-glucono- $\gamma$-lactone via 1,4-anhydro-2,3-isopropylidene4-thio-D-ribitol 14. ${ }^{22}$ These compounds differed in protecting groups that were used. When compound 15 was reacted with 6-chloropurine, PIFA, TMSOTf, and $\mathrm{Et}_{3} \mathrm{~N}$, quite surprisingly, compound 19 (54\%) was isolated with no trace of the desired $\mathbf{2 1 .}$

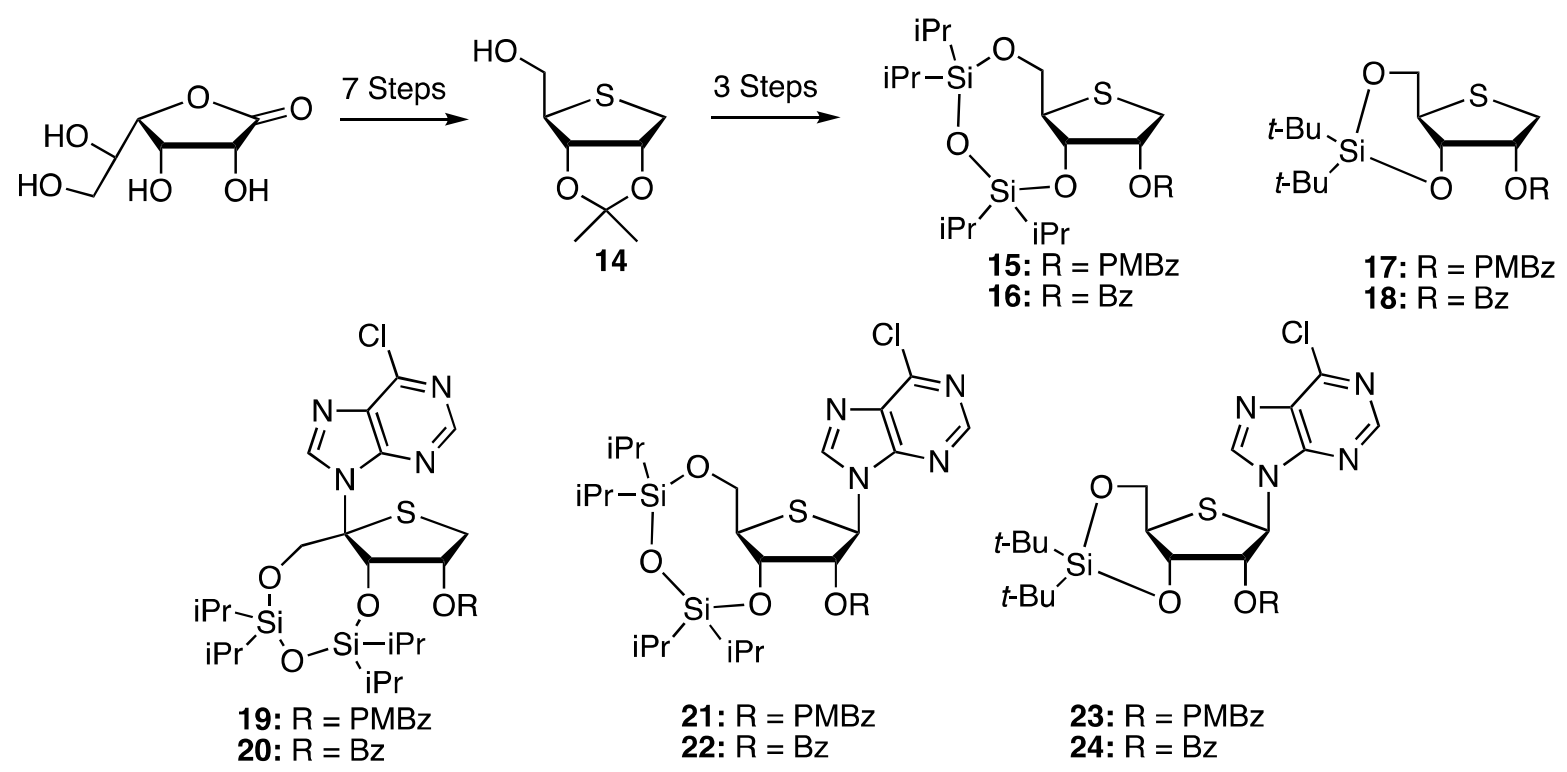

Scheme 4. Reactions of 6-chloropurine with differentially protected tetrahydothiophene derivatives.

On the other hand, the use of precursor $\mathbf{1 6}$ did lead to the desired $\mathbf{2 2}\left(16 \%+12 \%\right.$ of the $\mathrm{N}^{7}$ isomer) and about $5 \%$ of $\mathbf{2 0}$. But use of $\mathbf{1 7}$ and $\mathbf{1 8}$ both led to the desired products $\mathbf{2 3}\left(11 \%+17 \%\right.$ of the $\mathrm{N}^{7}$ isomer) and $\mathbf{2 4}$ $\left(22 \%+17 \%\right.$ of the $\mathrm{N}^{7}$ isomer), respectively. These data show that the reaction can partition between two cationic species (Figure 3). The authors proposed that in substrate 15, the PMBz group is less electron withdrawing than the $\mathrm{Bz}$ group in $\mathbf{1 6}$. Thus, the more acidic proton is removed, which in the case of $\mathbf{1 6}$ is at the less-substituted carbon atom, and this followed by capture by the purine at the desired center (see Scheme 1 for a mechanism). With substrates 17 and 18, they propose a significantly increased steric bulk at the silyl-protecting group, which then diverts deprotonation to the desired less-substituted carbon atom.
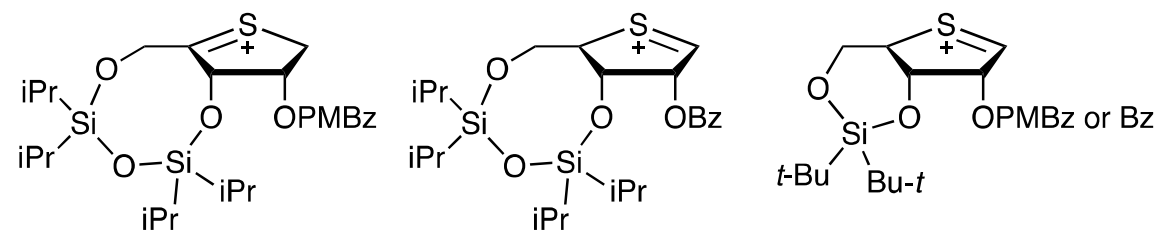

Figure 3. Possible thionium cations that are proposed in the reactions.

In order to test the hypothesis two compounds, 25 and 26, were synthesized from $\mathbf{1 4 . ~ T h e s e ~ w e r e ~}$ subjected to reactions with 6-chloropurine (Scheme 5). From 25, 44\% of $\mathbf{2 7}$ and $14 \%$ of 29 were obtained (in addition, $8 \%$ of the $\mathrm{N}^{7}$ isomer was formed). 


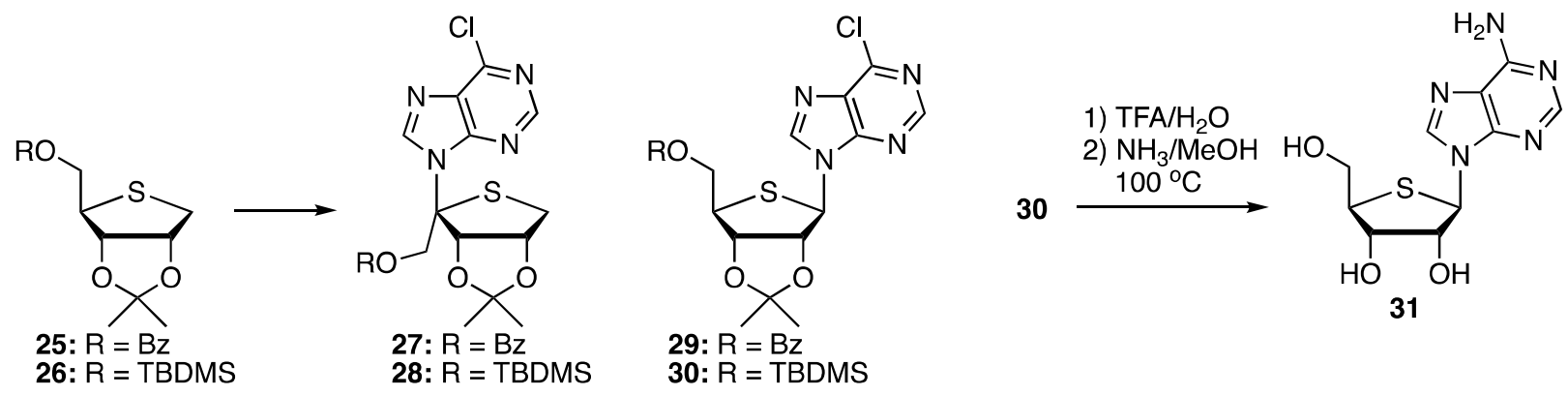

Scheme 5. Testing the hypothesis on the role of protecting groups on sites of reactions.

By contrast, in the reaction of $\mathbf{2 6}$ with 6-chloropurine, $<1 \%$ of $\mathbf{2 8}$ and $28 \%$ of $\mathbf{3 0}$ were obtained (along with $10 \%$ of the $\mathrm{N}^{7}$ isomer). This clearly showed that with 25 deprotonation occurred at the more-substituted carbon atom (more acidic proton was removed), whereas with 26, steric bulk directs deprotonation to the less-substituted carbon atom. Thioadenosine analogue $\mathbf{3 1}$ was then obtained by removal of the acetonide and silyl protection (66\%) and ammonolysis (90\%).

Another hypervalent iodine reagent, HTIB, has seen use in the synthesis of other thymidine nucleoside analogues. ${ }^{22}$ This chemistry in fact began with cis-2-butene-1,4-diol 1 (the precursor in Scheme 1) that was converted over 10 steps to the dibenzoyl thietane $\mathbf{3 2}$ (Scheme 6). Exposure of $\mathbf{3 2}$ to thymine, TMSOTf, Et ${ }_{3} \mathrm{~N}$, and $\mathrm{HTIB}$ in $\mathrm{CH}_{2} \mathrm{Cl}_{2}$ at $0{ }^{\circ} \mathrm{C}$ resulted in the five-membered nucleoside analogue 33 in $30 \%$ yield. Deprotection of 33 then gave product 34 (62\%).

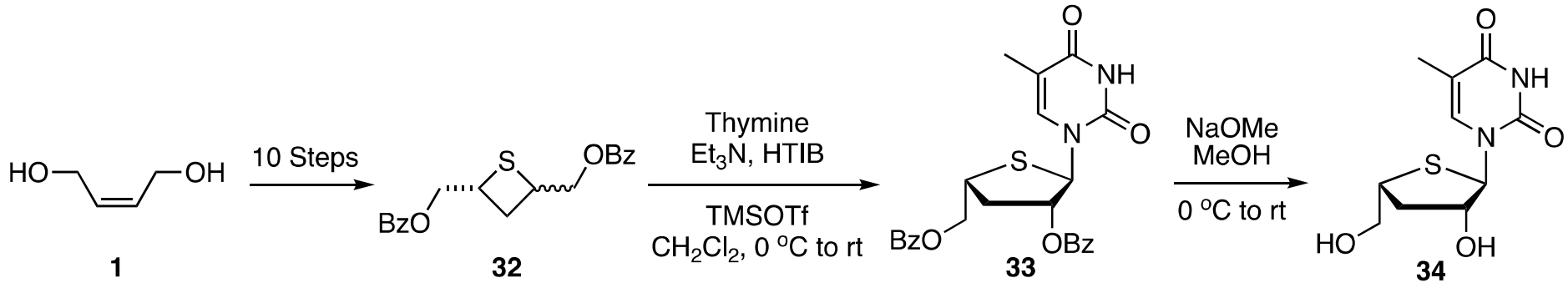

Scheme 6. Formation of a tetrahydrothiophene nucleoside analogue from a thietane.

Formation of product $\mathbf{3 3}$ rather than a thietane-derived product has been rationalized on the basis of neighboring group participation in the cis-isomer of precursor 32. This was supported by the isomerization shown in Scheme 7. On the basis of these observations, a proposed mechanism for the formation of $\mathbf{3 3}$ is shown in Scheme 7.

Just as with the synthesis of compound $\mathbf{4}$ (Scheme 2), a seleno analogue $\mathbf{3 5}$ was synthesized from D-ribose (Scheme $8, \mathrm{DMBz}=2,4$-dimethoxybenzoyl). ${ }^{23}$ This synthesis was beset with isomerization problems, analogous to those in Scheme 7 but resulting in a 6-membered product from a 5-membered precursor. Nevertheless, compound 35 was attainable. IOB, PIFA, PIDA, and HTIB were investigated for the "glycosylation" step, in conjuction with $\mathrm{Et}_{3} \mathrm{~N}$ or 2,6-lutidine as base. Best results were attained with 2 equiv. of uracil, 8 equiv. of TMSOTf, and 8 equiv. of 2,6-lutidine, in 1,2-dichloroethane at $50{ }^{\circ} \mathrm{C}$. Under these conditions a $64 \%$ yield of product 36 was obtained and $13 \%$ of 35 was recovered. 
TMSOTf

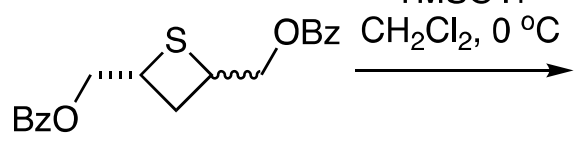

32

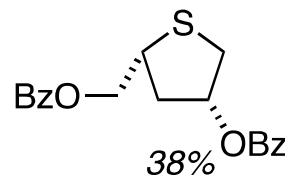

Mechanism:<smiles>O=C(O)C[C@H]1C[C@H](CO[18O])C1</smiles>

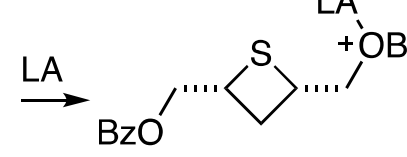

LA
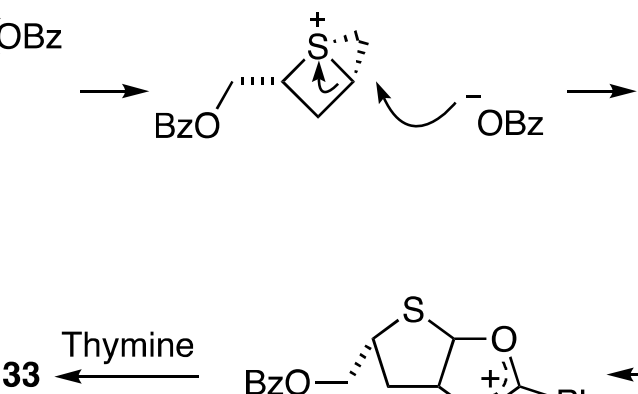

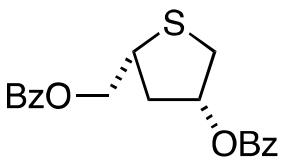

$\mathrm{OBz}$

$\mathrm{Et}_{3} \mathrm{~N}$

$33 \stackrel{\text { Thymine }}{\longleftarrow}$

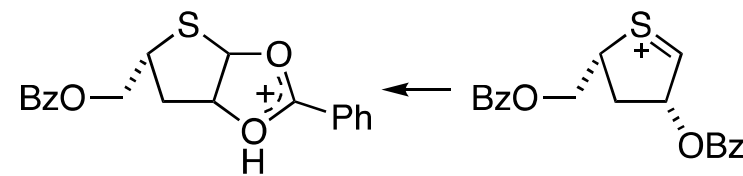

Scheme 7. Isomerization of cis-32 and a mechanism for the formation of product 33.

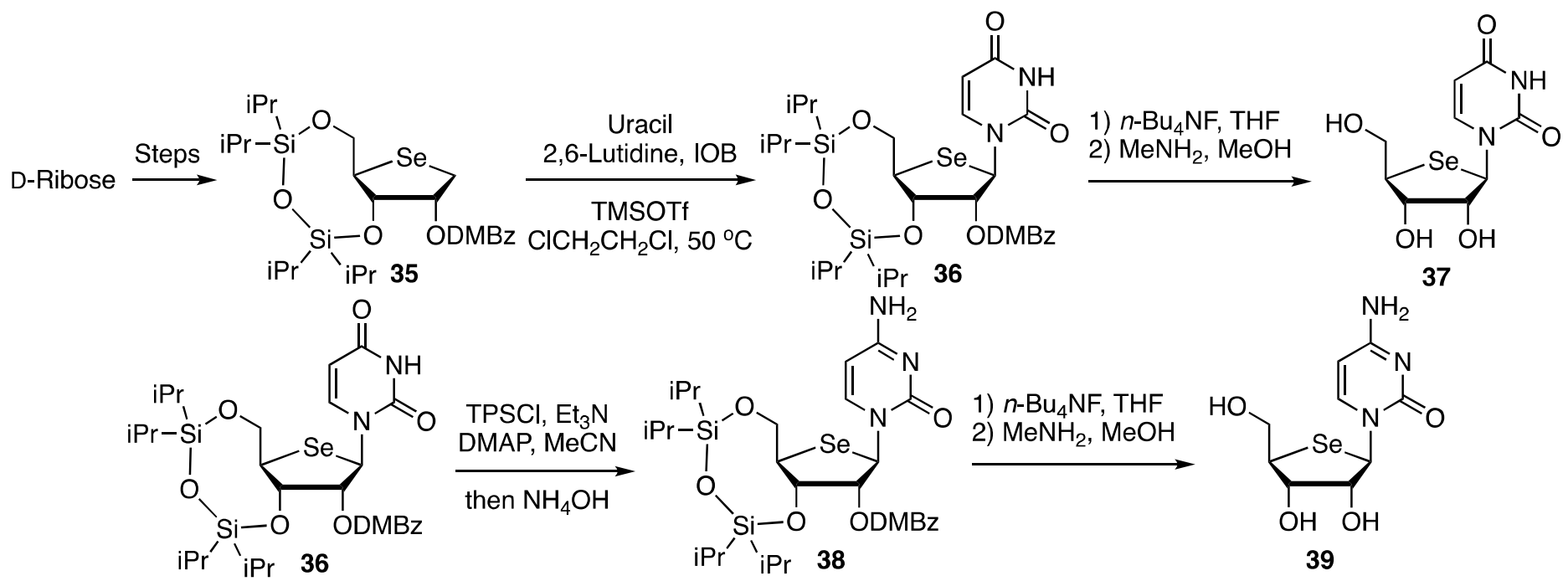

Scheme 8. Synthesis of seleno analogues of uridine and cytidine.

Deprotection of $\mathbf{3 6}$ gave the seleno analogue of uridine (37,66\%, Scheme 8). The amide group in $\mathbf{3 6}$ was converted to the sym-isopropylbenzene sulfonate, which was subjected to displacement with ammonia to yield compound 38 (91\%). Deprotection of this compound then gave the cytosine analogue 39 (64\%, Scheme 8).

Most recently, synthesis of the seleno analogues of purine nucleosides have been reported. ${ }^{24}$ Initially, the authors attempted syntheses with the selenosaccharide analogue 35 . However, this derivative was not useable; with 6-chloropurine the $\mathrm{N}^{7}$ "glycosylation" product was obtained with a hypervalent iodine reagent, but this could not be isomerized to the $\mathrm{N}^{9}$ isomer. On the other hand, reaction with 2-amino-6-chloropurine was unsuccessful. Thus, the authors pursued the use of $\mathbf{4 0}$, a variant of $\mathbf{2 6}$, that had previously been used in a Vorbrüggen coupling with the above-mentioned purines. ${ }^{25}$ In this work, the authors were able to isomerize the $\mathrm{N}^{7}$ "glycosylation" product to the $\mathrm{N}^{9}$ isomer, using TMSOTf (Scheme 9). 
As shown in Scheme 9, acetonide-protected $\mathbf{4 0}$ was reacted with 2 equiv. of silylated 6-chloropurine in the presence of IOB, TMSOTf, and 2,6-lutidine. A shorter reaction time led to lower amounts of the undesired $\alpha$ anomers but a lower reaction temperature led to a longer time for consumption of $\mathbf{4 0 . ~ H o w e v e r , ~ t h e ~}$ separated $\mathrm{N}^{7}$ isomer $\mathbf{4 2}$ was isomerized to $\mathbf{4 1}$ (overall 55\% yield from $\mathbf{4 0}$ ).

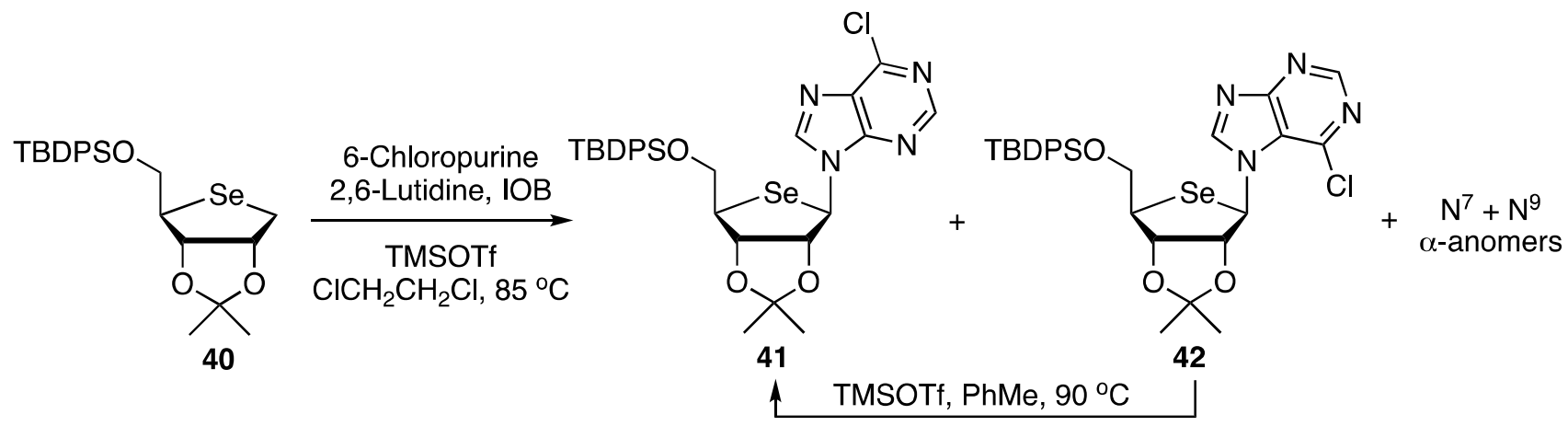

Scheme 9. Synthesis of the seleno analogue of 6-chloropurine riboside.

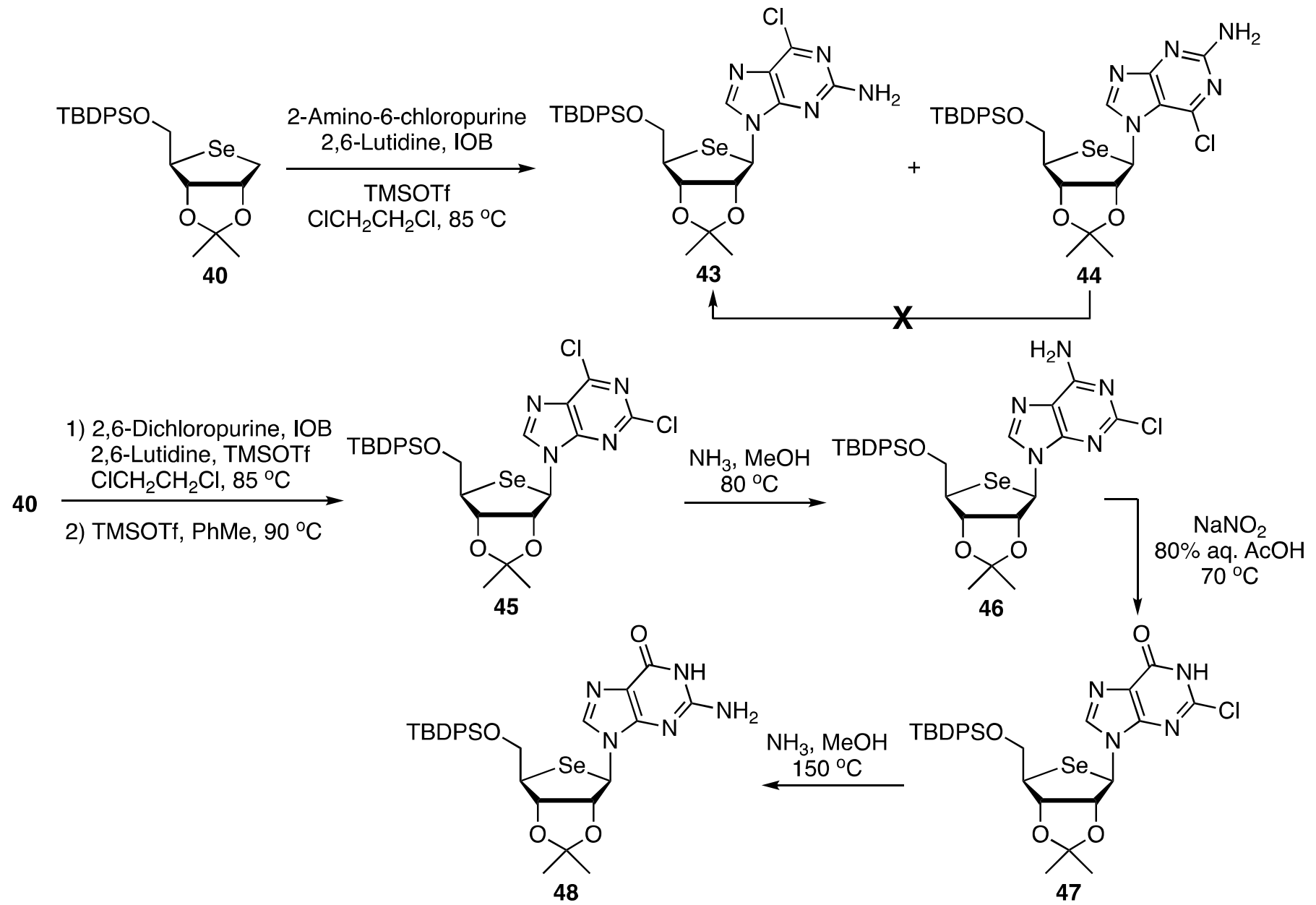

Scheme 10. Reactions of seleno sugar analogue $\mathbf{4 0}$ with 2-amino-6-chloro and 2,6-dichloropurines, and further transformations to a protected seleno guanosine analogue. 
Comparable chemistry was evaluated using 2-amino-6-chloropurine (Scheme 10). The desired $\mathrm{N}^{9}$ isomer (43) was a minor component (16\%) with the $N^{7}$ isomer (44) predominating in the product mixture (31\%). However, in this case, the $\mathrm{N}^{7}$ isomer could not be isomerized to the $\mathrm{N}^{9}$ derivative. In studies with other purine derivatives, the authors discovered that an amino group is a detriment to both yield and the isomerization.

In contrast to the outcome with 2-amino-6-chloropurine and other purines, reaction of 2,6-dichloropurine with 40 proceeded well to give an isomeric mixture that was readily isomerized to give 45 in $62 \%$ yield (Scheme 10). This compound was subjected to selective ammonolysis at the C6 position (46: 95\%) and diazotization/hydrolysis (47: 85\%). The protected seleno guanosine analogue $\mathbf{4 8}$ was finally obtained by ammonolysis at the $\mathrm{C} 2$ position, under more forcing conditions (77\%).

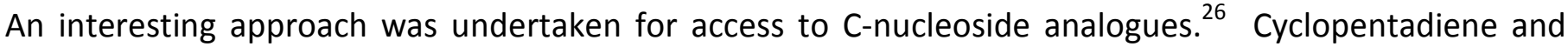
cyclohexadiene were subjected to hydrosilylation followed by conversion to the triethoxy and trimethyl silanes (Scheme 11). These compounds were investigated for their reactions with bis(trimethylsilyl)uracil, promoted by TMSOTf and a hypervalent iodine reagent (IOB, PIDA, PIFA, and HTIB, see Scheme 11). Of these, PIDA gave the best outcome.

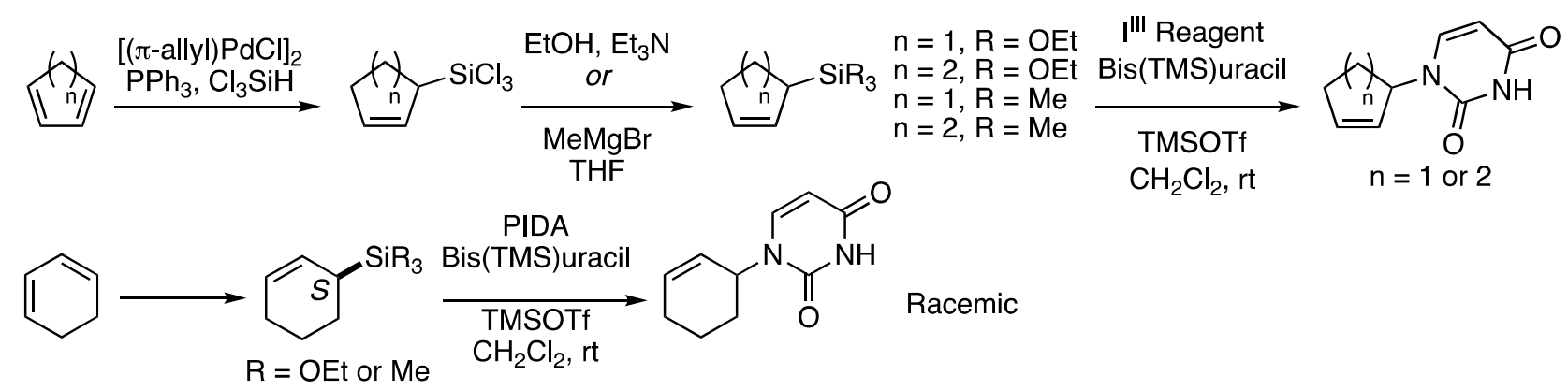

Scheme 11. Reactions of silyl derivatives of cyclopentene and cyclohexene with uracil.

Use of a chiral cyclohexene derivative returned a racemic product (Scheme 11). This indicated the formation of an allyl cation as a reactive intermediate that is captured by uracil. The next focus was the development of a synthesis of more substituted cyclohexene-based pyrimidine nucleoside analogues. For this, reaction of $(E)$-buta-1,3-dien-1-yltrimethylsilane was subjected to a [4 +2$]$ cycloaddition with dimethyl fumarate (Scheme 12). The $1: 1$ mixture of inseparable cycloadducts was reduced and silylated, resulting in separable $49 \mathrm{a}$ and $49 \mathrm{~b}$.
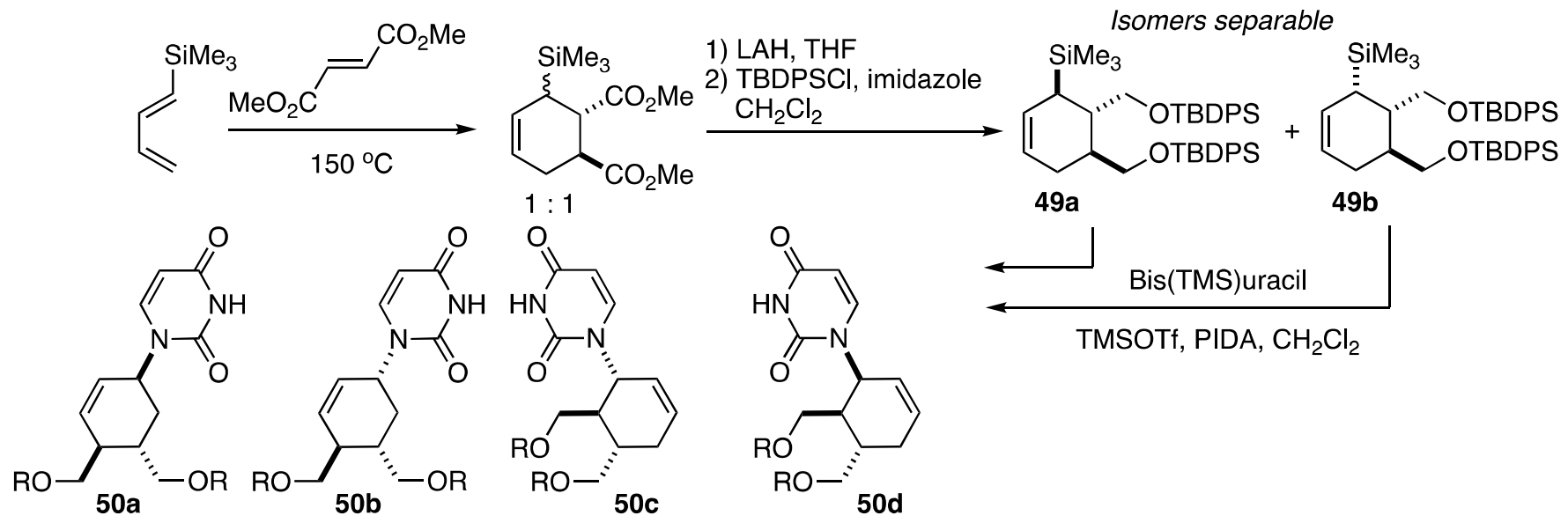

Scheme 12. Synthesis of cyclohexenyl uridine analogues. 
Each cyclohexene isomer (49a and $49 \mathrm{~b}$ ) was reacted with bis(trimethylsilyl)uracil, using PIDA, and TMSOTf. Isomer 49a reacted faster than $49 \mathrm{~b}$ and in the case of the latter, unreacted starting material (20\%) was reisolated. However, each precursor gave the four products 50a-d in different ratios. In order to separate the four products, the N3 position was benzoylated (51a-d in Scheme 13) and the product mixture was chromatographed. From this, compounds $\mathbf{5 1 a}$ and $\mathbf{5 1 d}$ were separated, whereas a mixture of $\mathbf{5 1 b}$ and $\mathbf{5 1 c}$ was obtained.

Debenzoylation of 51a-d gave $\mathbf{5 0 a - d}$ (two of which were separated). Sulfonylation of the amides in $\mathbf{5 0 a - d}$ gave 52a-d, displacement with ammonia gave the silyl-protected compounds 53a-d. At this stage, all four isomers were separable. Final desilylation with $n-\mathrm{Bu}_{4} \mathrm{NF}$ in THF gave the four cytidine analogues 54a-d.

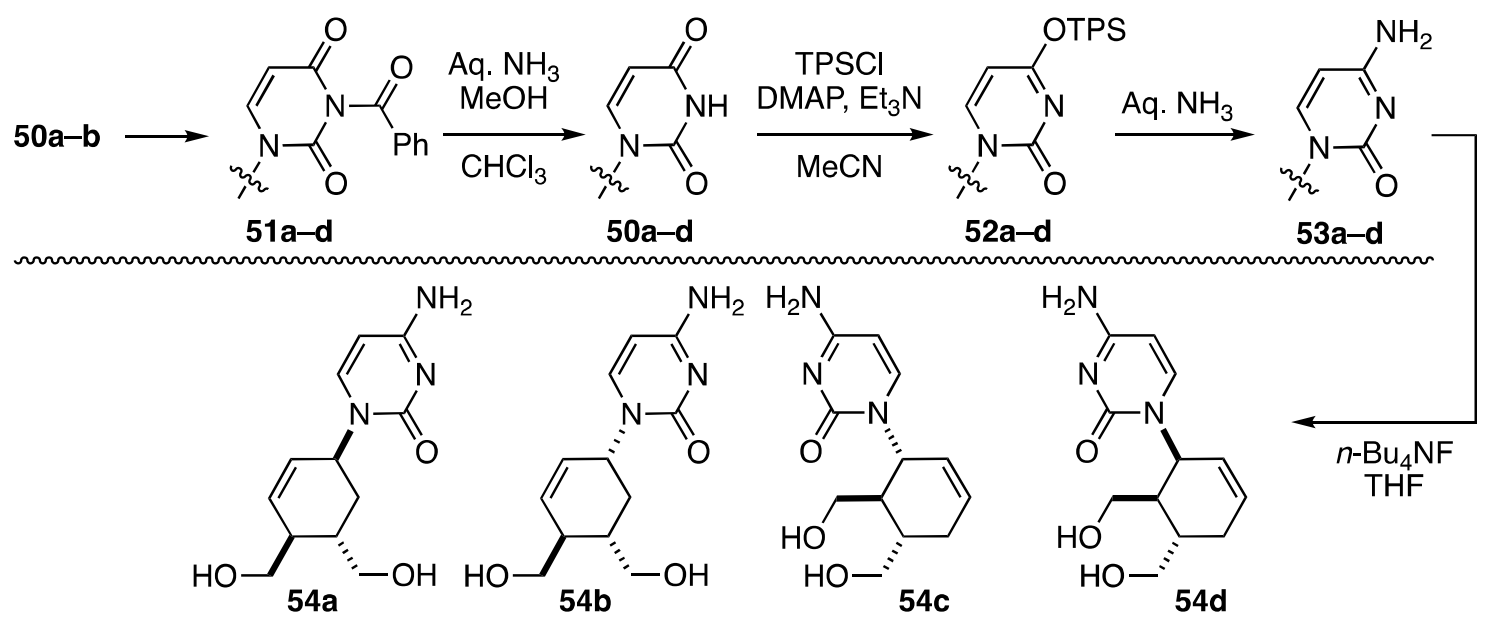

Scheme 13. Synthesis of four isomeric cytidine nucleoside analogues.

In 2012, a method to access oxygen-containing uracil nucleoside analogues was reported. ${ }^{27}$ In this work, initially reactions of dihydropyran and dihydrofuran with bis(trimethylsilyl)uracil were assessed. Reaction of dihydropyran using 2 equiv. of bis(trimethylsilyl)uracil, 1.5 equiv. of PIDA, and 0.4 equiv. of TMSOTf (-40 ${ }^{\circ} \mathrm{C}$ to rt), gave a $31 \%$ yield of product 55 (Scheme 14). The same product was also obtained in $24 \%$ yield upon using 1.5 equiv. of PIDA and 0.2 equiv. of $\mathrm{Cu}(\mathrm{OTf})_{2}$, at rt. On the other hand, reaction of dihydrofuran with 2 equiv. of bis(trimethylsilyl)uracil, 1.5 equiv. of PIDA, and 0.4 equiv. of TMSOTf $\left(0{ }^{\circ} \mathrm{C}\right.$ to $\mathrm{rt}$ ), gave three products (5658), shown in Scheme 14.

A proposed mechanism is shown in Scheme 14, where an initial addition product from dihydropyran undergoes reaction with bis(trimethylsilyl)uracil (either directly or via an oxocarbenium ion), followed by an elimination to yield 55. Alternatively, a double elimination from the initial addition product can produce a 2,3dihydropyrylium ion that can undergo capture to yield $\mathbf{5 5}$. With dihydrofuran, product 56 can be obtained by elimination, as was the case with 55. But here, a rearrangement of the acetate can yield 57, while involvement of the amide carbonyl in the departure of $\mathrm{Phl}$ and $\mathrm{AcO}^{-}$can yield cyclized product 58.

Next the oxidative coupling of enol ethers with bis(trimethylsilyl)uracil was studied using 1 equiv. each of the protected uracil, (PhSe) 2 , PIDA, and 0.1 equiv. of TMSOTf. Reactions of dihydrofuran and dihydropyran were conducted at $\mathrm{rt}$, that of ethyl vinyl ether at $-5{ }^{\circ} \mathrm{C}$, and those of the substituted dihydropyrans at $-20{ }^{\circ} \mathrm{C}$. Products from these reactions are shown in Scheme 15 as also a plausible mechanism for the reaction. From among the products, oxidation of compound 59 with $m$-CPBA in $\mathrm{CH}_{2} \mathrm{Cl}_{2}$ followed by exposure to pyridine in PhMe at $50{ }^{\circ} \mathrm{C}$, gave compound 55 in an overall $60 \%$ yield. 

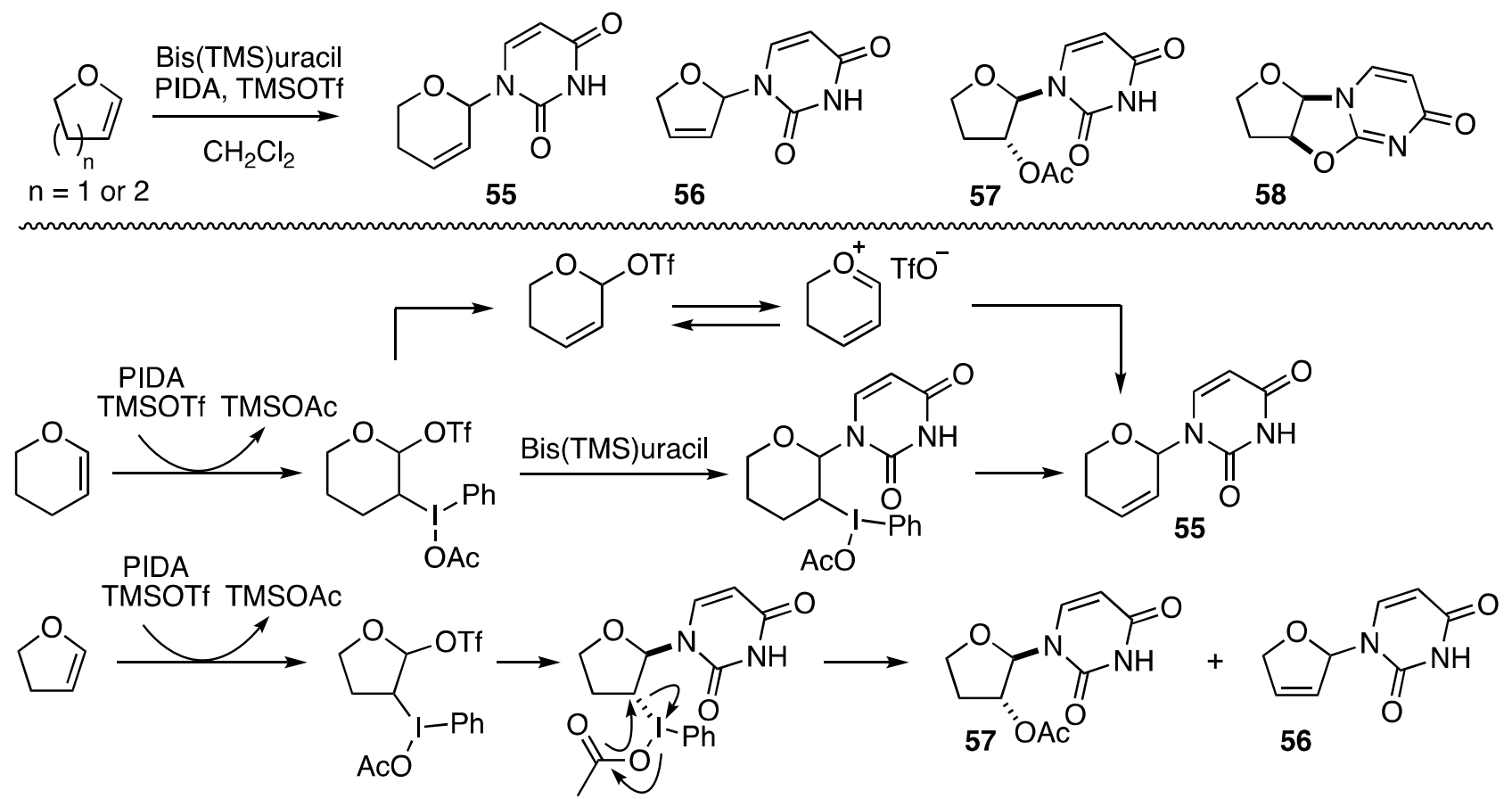

Scheme 14. Reactions of dihydropyran and dihydrofuran with bis(trimethylsilyl)uracil.
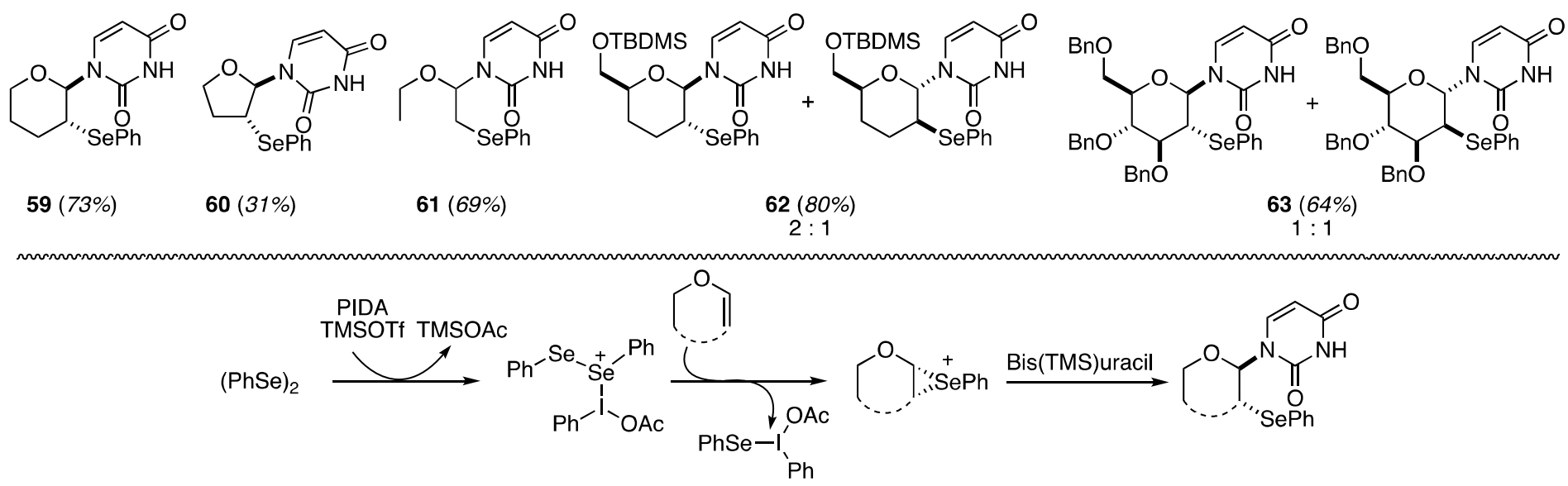

Scheme 15. Various uracil derivatives prepared via the "seleno-glycosylation" approach and a possible mechanism.

The chemistry described above was then applied to the synthesis of dihydropyranyl uracil and cytosine nucleoside analogues. ${ }^{28}$ For this, compound 64 was synthesized over 6 steps from cis-2-butene-1,4-diol 1 (Scheme 16). Reaction of 64 with 1 equiv. each bis(trimethylsilyl)uracil, (PhSe)2, PIDA, and 0.1 equiv. of TMSOTf, gave a 2 : 1 ratio of diastereomers $65 \mathrm{a}$ and $65 \mathrm{~b}$ in $51 \%$ yield. The former was anticipated as the major product based on a conformational analysis of anticipated intermediates. These diastereomers could not be separated either after elimination $(66 a+66 b, 71 \%$ over 2 steps) or after deprotection at rt $(67 a+67 b$, quantitative). 


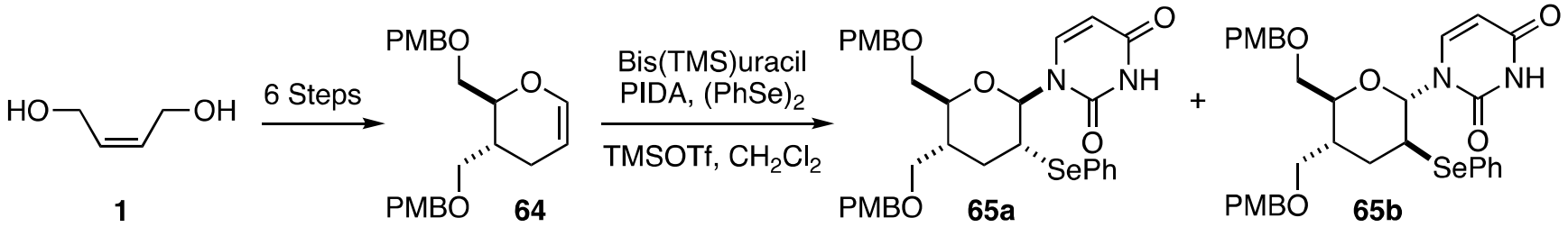

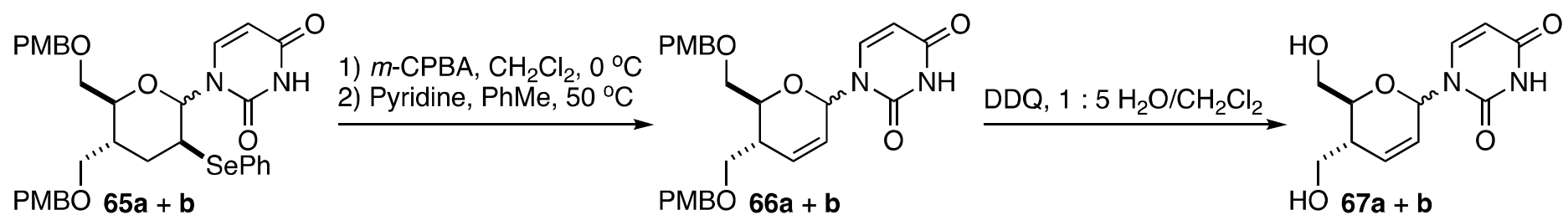

Scheme 16. Synthesis of dihydropyranyl uracil nucleoside analogues.

Acetylation of the hydroxyl groups of $\mathbf{6 7}$ gave chromatographically separable isomers $\mathbf{6 8 a}$ and $\mathbf{6 8 b}$ (Scheme 17). The amide groups in each isomer were converted to the sym-triisopropylphenylsulfonates and subjected to ammonolysis. Finally, the acetate protecting groups were removed with ammoniacal methanol (yield of $70 \mathrm{a}=60 \%$ over two steps and that of $70 \mathrm{~b}=61 \%$ over two steps).

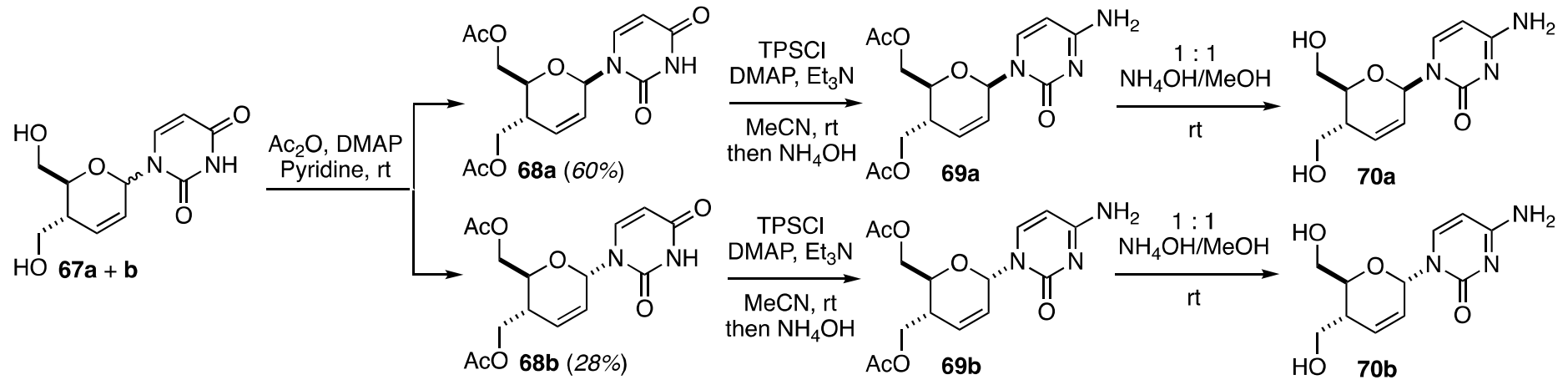

Scheme 17. Separation of the uracil nucleoside analogues and conversion to the cytosine derivatives.

\section{Miscellaneous Hypervalent lodine Reagent Mediated Nucleoside Modifications}

In 1998, PIFA was utilized to introduce SPh and SePh groups into uracil derivatives and uridine analogues. ${ }^{29}$ The mechanism of this reaction (Scheme 18) is related to that shown in Scheme 15 and the reaction provides good yields of the products (Scheme 18). For sulfenylation the ratio of nucleosides/(PhS) $2 / \mathrm{PIFA}=1: 1: 2$, whereas for selenation it was $1: 0.7: 0.7$.

It should be noted that $\mathrm{PIDA} / \mathrm{Br}_{2}$ and $\mathrm{PIDA} / \mathrm{I}_{2}$ have been used to halogenate pyrimidine derivatives at the C5 position, in $86-94 \%$ yields. $^{30}$ However, details are not provided herein, as the chemistry has not been applied to nucleosides or nucleoside analogues. 


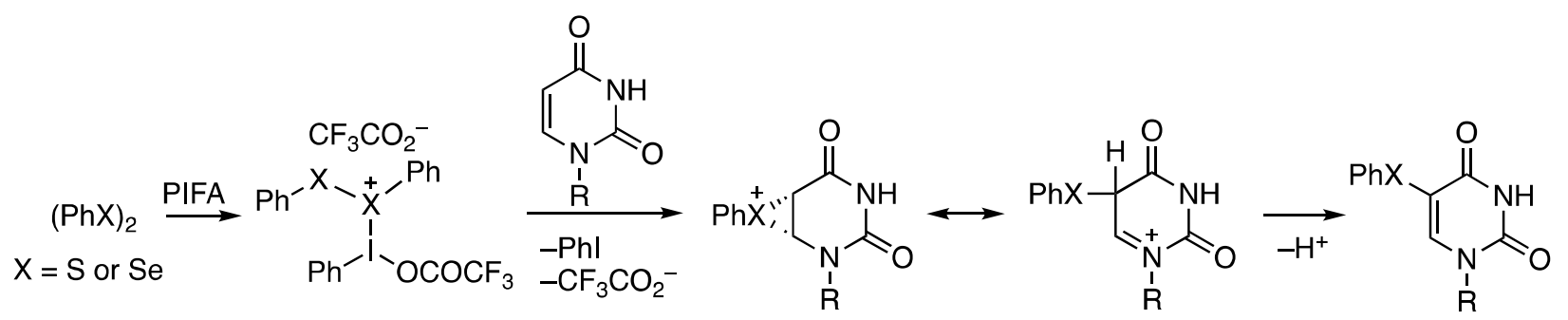<smiles>[R]OC[C@H]1O[C@H](n2cc([Y])c(=O)[nH]c2=O)[C@H]([R])[C@@H](O)[C@@H]1O</smiles>

71a: $X=S ; R=A c(82 \%)$

71b: $X=S e ; R=A c(97 \%)$

71c: $X=S ; R=B z(95 \%)$

71d: $X=S e ; R=B z(95 \%)$

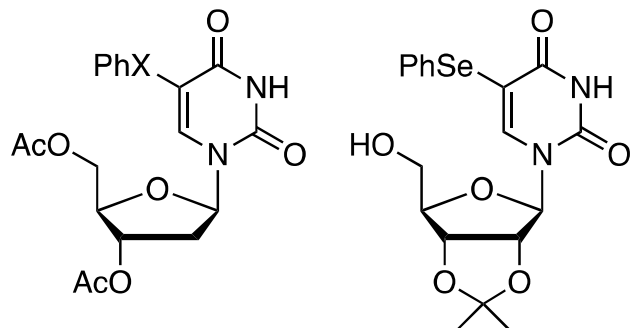

$73(99 \%)$<smiles>O=C(OCC(COC(=O)c1ccccc1)OCC(=O)c1ccccc1)Oc1ccccc1</smiles>

$74(97 \%)$<smiles>[X]c1cn(COCCOC([R])C)c(=O)[nH]c1=O</smiles>

75a: $X=S(80 \%)$

75b: $X=\operatorname{Se}(94 \%)$

Scheme 18. Sulfenylation and selenation of uracil derivatives and uridine nucleosides, and analogues.

Oxidation of oximes with hypervalent iodine reagents leads to nitrile oxides that can participate in [3+2] cycloadditions (Scheme 19). ${ }^{31}$ Such chemistry has been utilized to prepare a set of isoxazole-functionalized nucleoside analogues, circumventing the need for the isolation and manipulations of hydroximoyl chlorides.

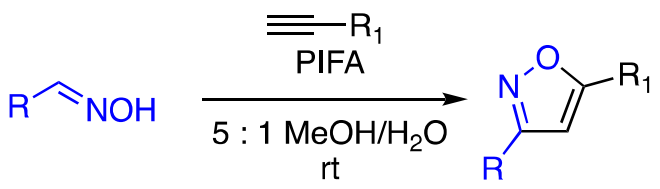

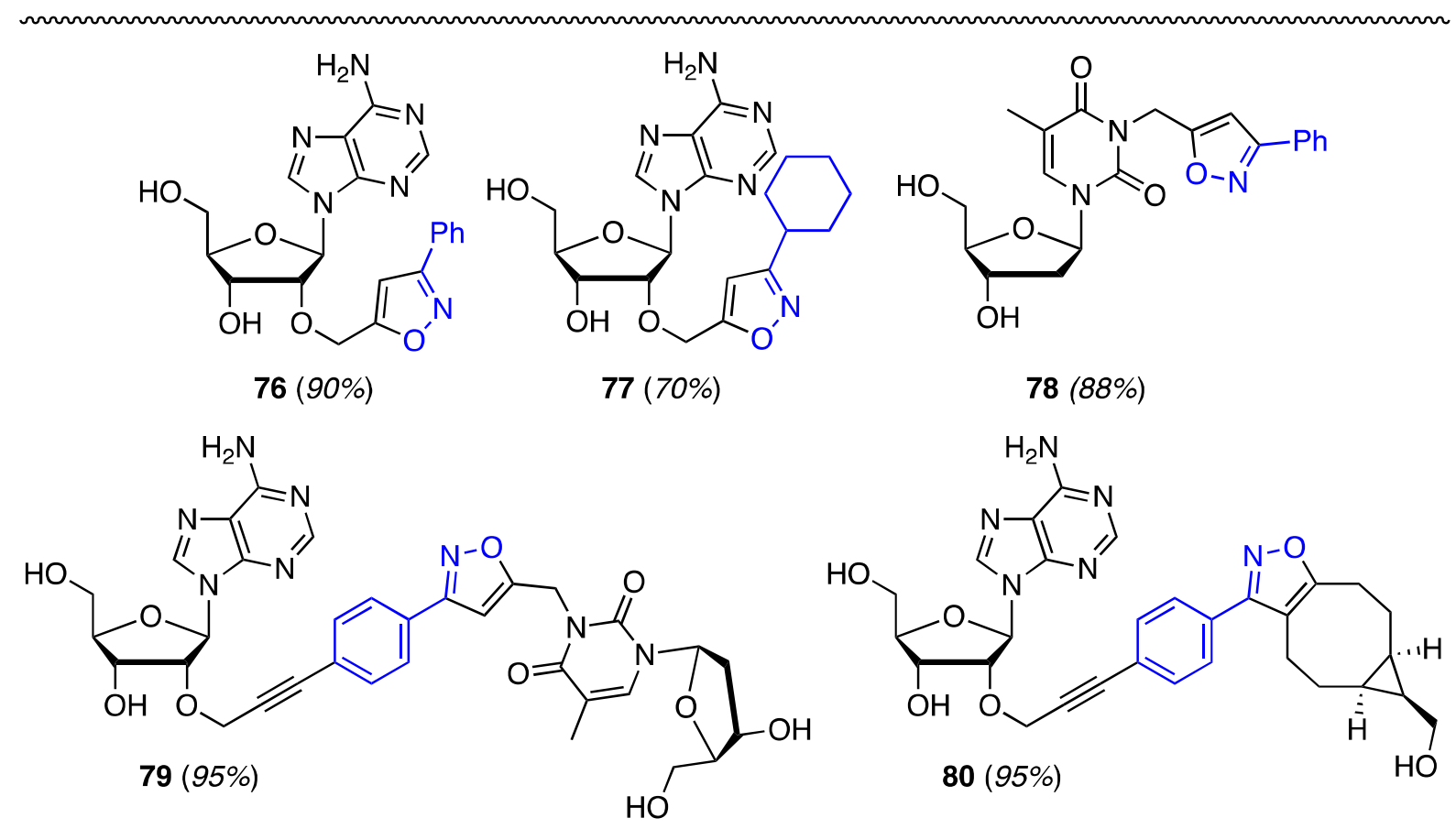

Scheme 19. Use of PIFA for the cycloaddition of nitrile oxides with alkynes. 
In 2016, a similar oxime oxidation was utilized to prepare purine derivatives with an appended isoxazole moiety. The precursor, (6-piperidin-1-yl)acetaldehyde was synthesized by reaction of 6-piperidin-1-yl-9Hpurine with 2-bromoacetaldehyde diethyl acetal, with $\mathrm{K}_{2} \mathrm{CO}_{3}$ in $\mathrm{DMF}$ at $140{ }^{\circ} \mathrm{C}$ ( $\mu$ wave irradiation, 45 min, $83 \%$ ). This compound was hydrolyzed to the aldehyde with $\mathrm{HCl}$ that was converted to the oxime 81 by treatment with $\mathrm{HONH}_{2} \cdot \mathrm{HCl}$ in $\mathrm{EtOH}$, in the presence of $\mathrm{KOAC}$ at $80{ }^{\circ} \mathrm{C}(2 \mathrm{~h}, 86 \%)$. Reaction of 81 with propargylic alcohols yielded the corresponding isoxazole appended purine derivatives (Scheme 20). A phosphate derivative of propargyl alcohol also reacted (83).
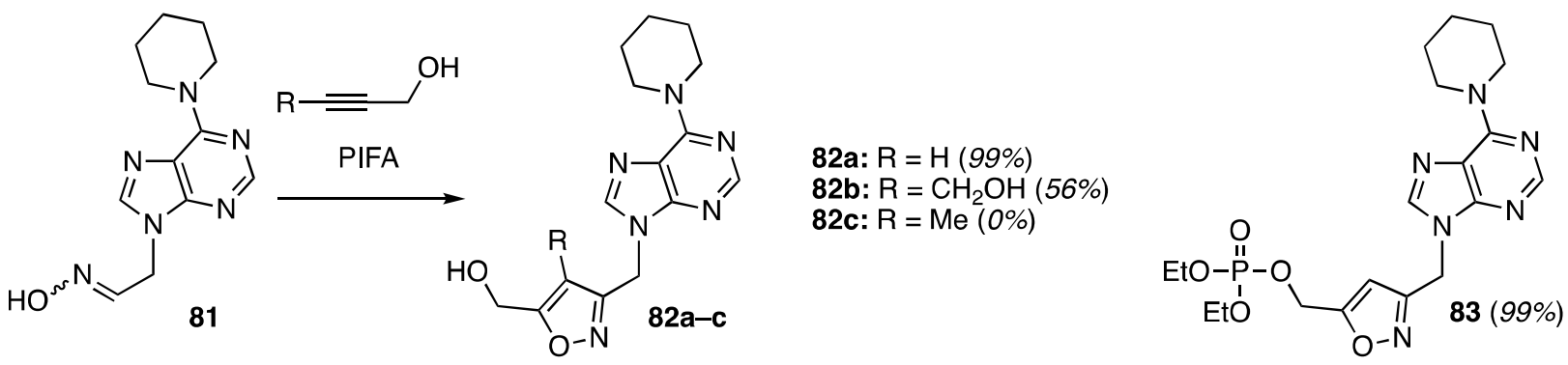

Scheme 20. Formation of isoxazolyl 6-pyrrolidinyl purine derivatives by PIFA oxidation.

This chemistry was also applied to the synthesis of isoxazoline-modified 6-pyrrolidinyl purine nucleoside analogues. Best conditions involved the use of 10 equiv. of the olefinic alcohols or unsaturated phosphate esters, 1.5 equiv. of PIFA, and short reaction times (5-10 min). Some representative examples that were synthesized are shown in Figure 4.

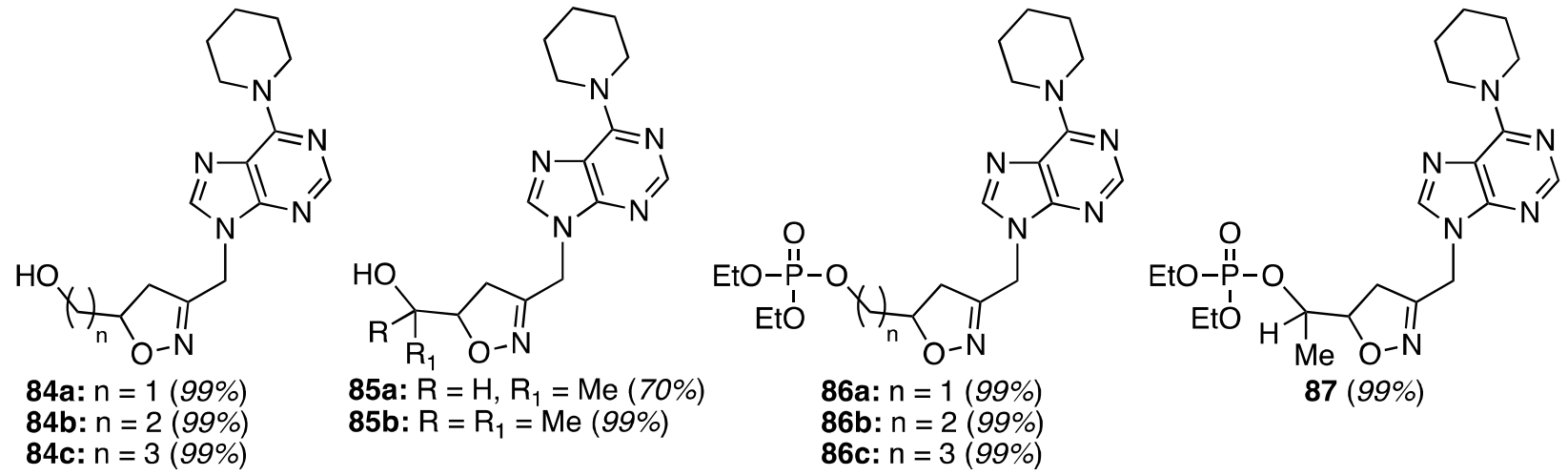

Figure 4. Examples of isoxazoline-substituted 6-pyrrolidinyl purine derivatives via PIFA oxidation.

\section{Purinyl N-Directed C-H Bond Oxidation Using PIDA}

In 2011, efforts by others ${ }^{33}$ and by us $^{34}$ led to the knowledge that the embedded purinyl nitrogen atoms can be used to direct $\mathrm{C}-\mathrm{H}$ bond activation at remote sites in nucleosides. Both $\mathrm{Pd}(\mathrm{OAc})_{2}$ and $\left.(\mathrm{ArRuCl})_{2}\right)_{2}$ catalysts were effective. The former was useable for ribonucleosides whereas the latter was more generally applicable across ribo and deoxyribonucleosides.

On the basis that the purinyl nitrogen atom appeared capable of directing a remote $\mathrm{C}-\mathrm{H}$ bond activation, a single example was published in 2011 that showed that 6-phenylpurine ribonucleoside, protected as a 
triacetate, could be oxidized in the aryl ring (Scheme 21, in this work, several purine derivatives were evaluated as well). ${ }^{35}$ The reaction gave mono and diacetoxylated products in a $2.1: 1$ ratio (77\% yield).

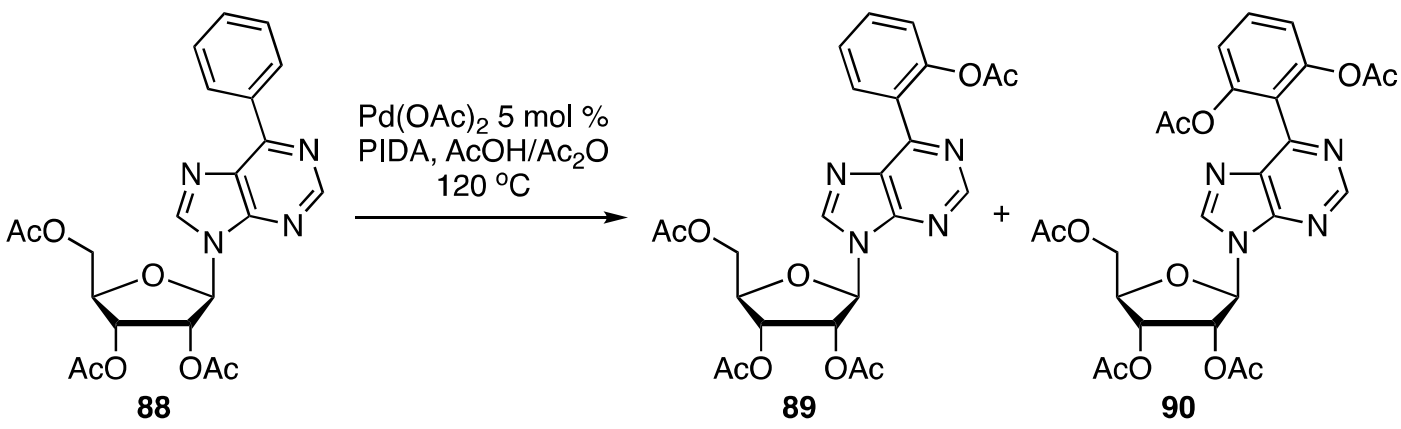

Scheme 21. Acetoxylation of an acetate-protected 6-phenyl purine ribonucleoside.

We had commenced our own independent studies on this bond oxidation at about the same time, but we had focused on the more labile 2'-deoxyribonucleosides. ${ }^{36}$ It has recently been quantitatively shown that 2'deoxyribonucleosides are substantially more labile as compared to the ribosyl counterparts. For example, the $t_{1 / 2}$ for the deglycosylation of adenosine in $0.1 \mathrm{M} \mathrm{HCl}$ is 11 days at $37{ }^{\circ} \mathrm{C}$. By contrast, the $t_{1 / 2}$ for deglycosylation of $2^{\prime}$-deoxyadenosine is $15 \mathrm{~min}^{37}$
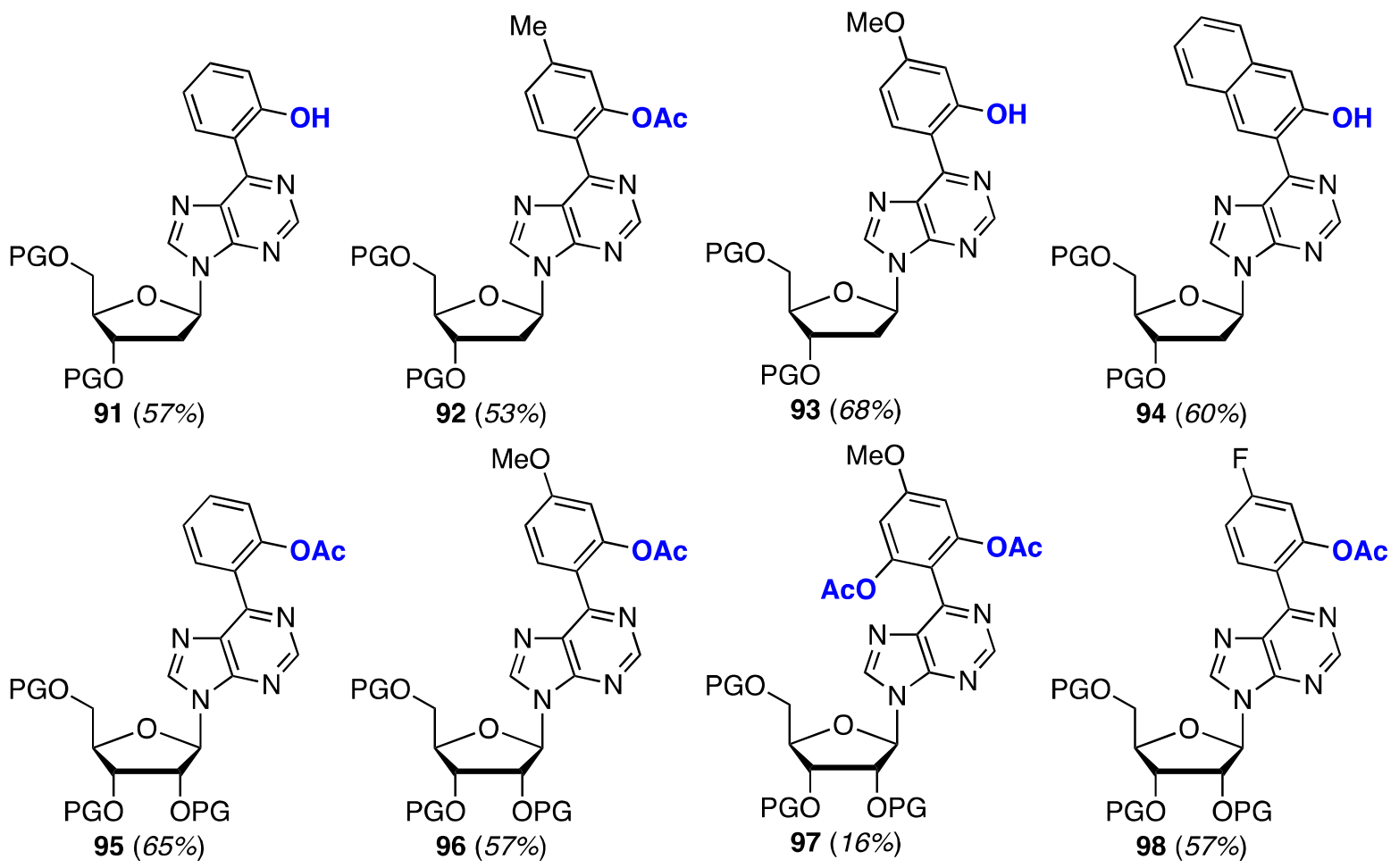

Figure 5. Oxidation of 6-aryl purine nucleosides with $\mathrm{Pd}(\mathrm{OAc})_{2}$ and $\mathrm{PIDA}\left(\mathrm{PG}=t-\mathrm{BuMe} \mathrm{Si}_{2}\right.$.

In our investigations, we initially screened conditions for the $\mathrm{C}-\mathrm{H}$ bond activation and oxidation using both 9-benzyl-6-phenyl-9H-purine and 6-phenyl purine 2'-deoxyribonucleoside with $t$-BuMe ${ }_{2} \mathrm{Si}$ protecting groups on the saccharide. Whereas use of 5 or $10 \mathrm{~mol} \% \mathrm{Pd}(\mathrm{OAc})_{2}$ with 1.2 equiv. of PIDA, in $\mathrm{MeCN}$ at $100{ }^{\circ} \mathrm{C}$, gave identical product recoveries with the purine, use of 5 mol\% $\mathrm{Pd}(\mathrm{OAc})_{2}$ and 1.2 equiv. of PIDA gave incomplete 
reaction with the nucleoside. On the other hand, 10 mol\% of $\mathrm{Pd}(\mathrm{OAc})_{2}$ and 1.5 equiv. of PIDA, in MeCN at 100 ${ }^{\circ} \mathrm{C}$, gave a $57 \%$ yield of a monohydroxylated product (partial loss of the acetate was observed and so the product was treated with $\mathrm{NaOMe} / \mathrm{MeOH}$ to fully hydrolyze the ester). As expected, the use of conditions reported in reference 35 yielded no product. Using this method, several hydroxylated nucleosides were prepared (Figure 5).

When partial hydrolysis of the ester was observed, the final product was obtained after exposure to $\mathrm{NaOMe} / \mathrm{MeOH}$. Reaction of the $p$-tolylphenyl precursor to 92 was conducted with 0.85 equiv. of PIDA as some undesired products were observed when 1.5 equiv. was employed. Reaction of the $p$-methoxyphenyl precursor gave mono and diacetoxylation products in a $3.6: 1$ ratio.
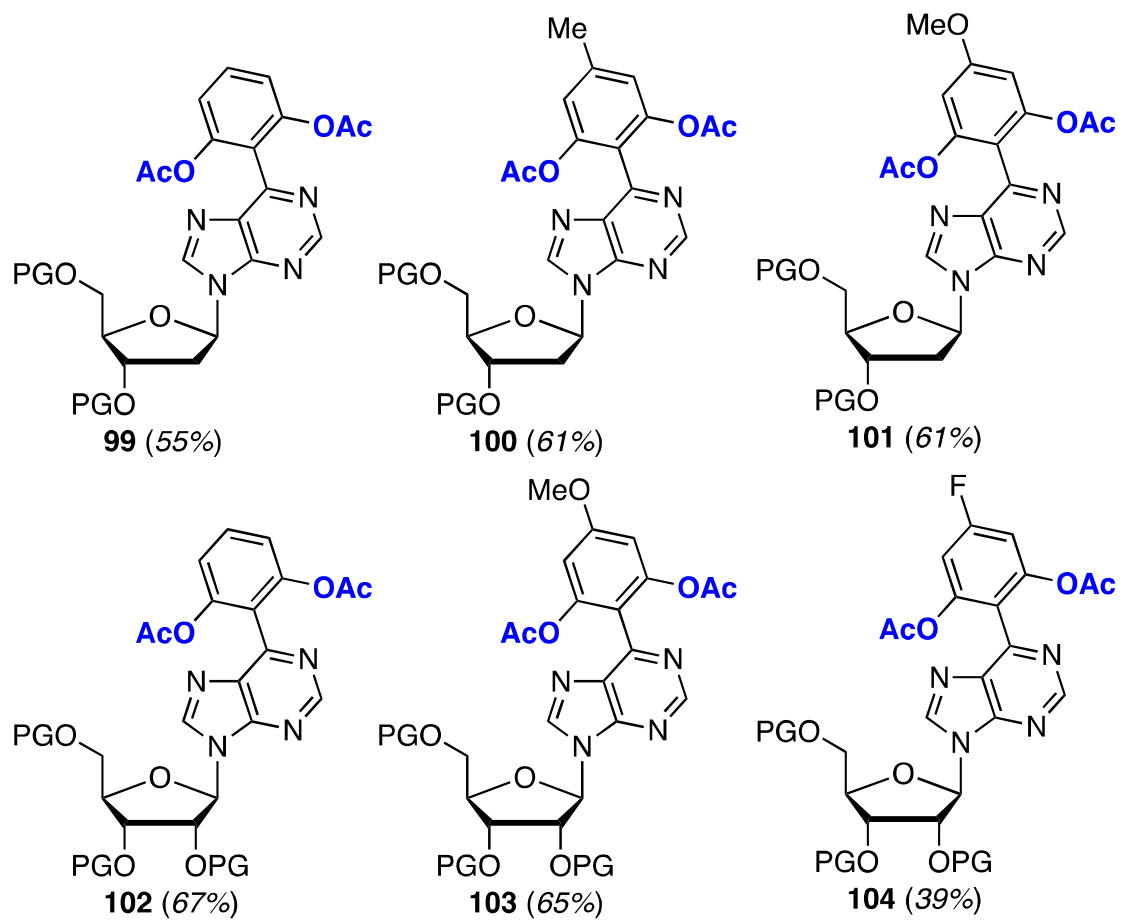

Figure 6. Diacetoxylation of 6-aryl purine nucleosides with $\mathrm{Pd}(\mathrm{OAc})_{2}$ and PIDA (PG $\left.=t-\mathrm{BuMe} \mathrm{S}_{2} \mathrm{Si}\right)$.

6-Aryl purine 2'-deoxy and ribonucleosides could also be dihydroxylated by using $15 \mathrm{~mol} \%$ of $\mathrm{Pd}(\mathrm{OAc})_{2}$ and 3 equiv. of PIDA. In all cases tested, the acetoxy group was not hydrolyzed. Reaction of the $p$ fluorophenyl precursor gave only $39 \%$ of the bis-oxidized product but $32 \%$ of the mono-acetoxylated product (98) was isolated from this reaction.

In the course of these investigations a dimeric cyclopalladated intermediate $\mathbf{1 0 5}$ was obtained from the reaction of a 6-(2-naphthalenyl)purine precursor (Scheme 22). This compound was a reasonable precatalyst for the acetoxylation of an arylpurine and a 6-arylpurine nucleoside, in the presence of PIDA. The crystallographically determined Pd-Pd bond distance in 105 was $2.844 \AA$. This is comparable to the bond distances in cyclopalladated dimers obtained from 2-phenylpyridine (2.862 $\AA$ ) and 2-p-tolylpyridine (2.857 $\AA))^{38}$ and benzo[ $\left.h\right]$ quinoline $(2.84 \AA \AA) .{ }^{39}$ Thus, Pd-Pd interaction may be important for the formation of a Pd $d^{11 !}-$ $\mathrm{Pd}^{\text {III }}$ bridged dimer 106, and species such as these are implicated in $\mathrm{N}$-directed $\mathrm{C}-\mathrm{H}$ bond oxidations. ${ }^{39,40}$ 

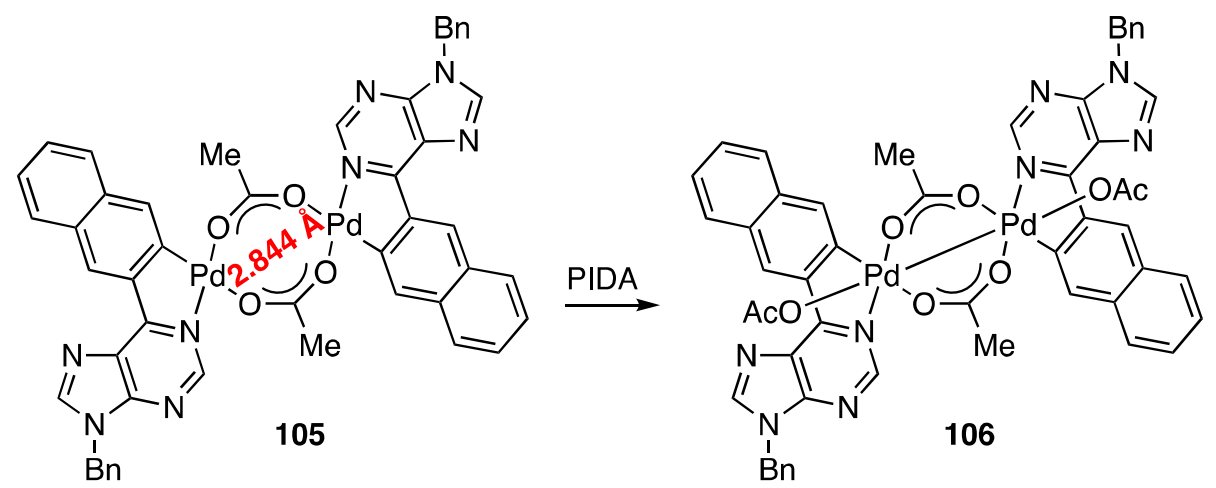

Scheme 22. An isolated and cystallographically characterized cyclopalladated, $\mathrm{Pd}$ "-Pd" dimer, and its potential conversion to a Pd"II-Pd"I species by PIDA.

\section{Cu-Catalyzed Intramolecular C-N Bond Formation in Purine Nucleosides Using PIDA}

In 2012, PIDA and other oxidants (oxone, $\mathrm{Fe}_{2}\left(\mathrm{NO}_{3}\right)_{2} \cdot 9 \mathrm{H}_{2} \mathrm{O}, 1$,4-benzoquinone, and $\mathrm{K}_{2} \mathrm{~S}_{2} \mathrm{O}_{8}$ ) were evaluated in combination with copper salts $\left(\mathrm{Cul}, \mathrm{CuBr}, \mathrm{Cu}(\mathrm{OAc})_{2}\right.$, and $\left.\mathrm{Cu}(\mathrm{OTf})_{2}\right)$ for the cyclization of $\mathrm{N}^{6}$-aryladenosine derivative, protected with acetyl groups on the sugar, to benzimidazolyl purine nucleoside analogues. ${ }^{41} 1.5$ Equiv of PIDA and $5 \mathrm{~mol} \%$ of $\mathrm{Cu}(\mathrm{OTf})_{2}$ in $1: 1 \mathrm{AcOH} / \mathrm{Ac}_{2} \mathrm{O}$, at $80{ }^{\circ} \mathrm{C}$, proved to be optimal conditions. Using these conditions several benzimidazopurine ribonucleoside analogues were prepared (Scheme 23). It appears that the presence of a methyl or methoxy group at the ortho position to the amino group caused a reduction in the yield, as also did a naphthyl group.
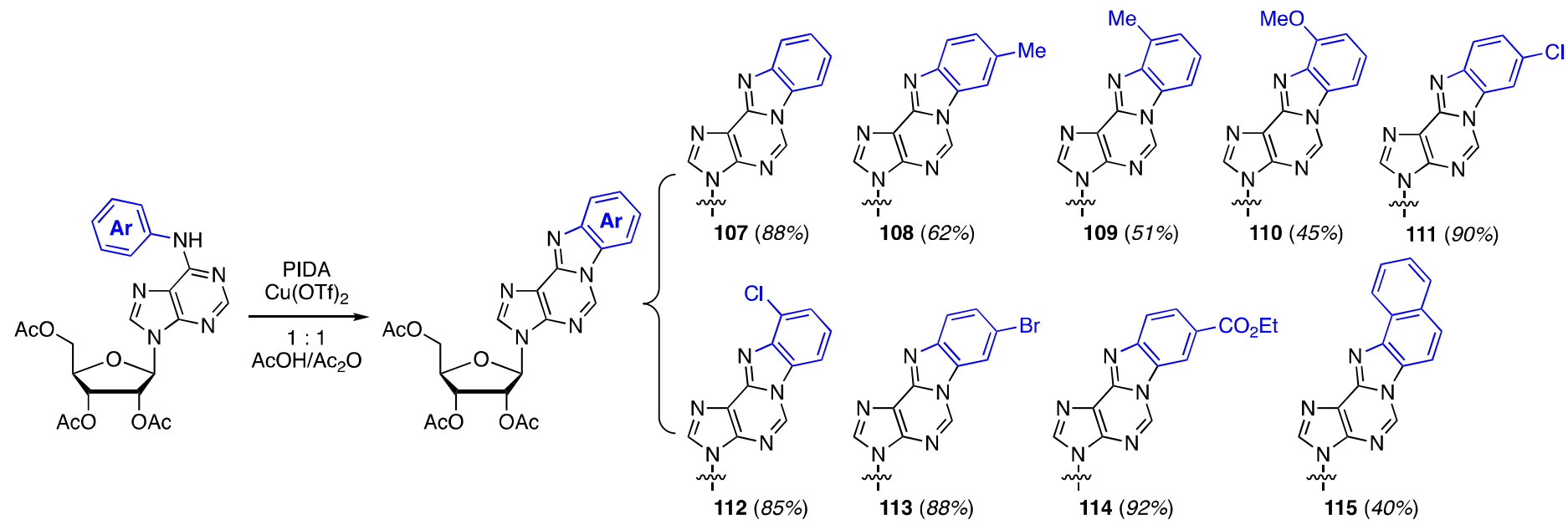

Scheme 23. PIDA/Cu(OTf $)_{2}$-mediated cyclization of $\mathrm{N}^{6}$-aryl adenosine triacetate derivatives to benzimidazopurine nucleoside analogues.

The mechanism of this reaction (Scheme 24) proposes a purinyl $N^{1}$ atom-directed cupration of the aryl ring, possibly via the tautomer. This would be followed by a reductive elimination, with PIDA reoxidizing the copper catalyst. 


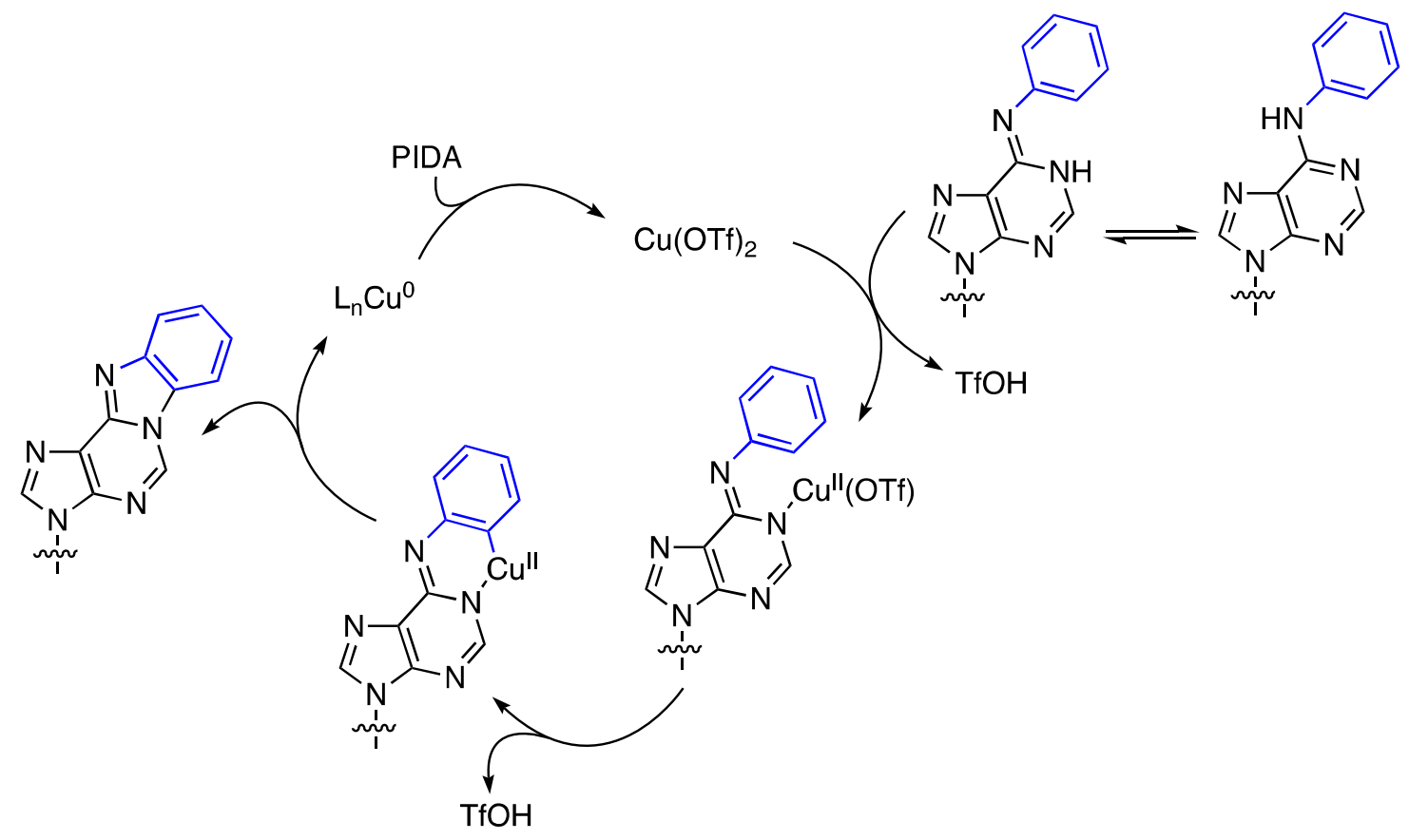

Scheme 24. A possible catalytic cycle for the $\mathrm{Cu}(\mathrm{OTf})_{2} / \mathrm{PIDA}-$ mediated cyclization

\section{Metal-Free Intramolecular C-N Bond Formation in Purine Nucleosides Using PIDA}

As with the $\mathrm{C}-\mathrm{H}$ bond oxidation chemistry, we were intrigued about the applicability of such a cyclization reaction to 2'-deoxyribonucleosides. As mentioned earlier, the 2'-deoxyribonucleosides are anticipated to be much more labile than the ribosyl counterparts, particularly those stabilized by electron-withdrawing acetates. We began our investigations by applying the previously described conditions ${ }^{41}$ to silyl-protected $N^{6}$-phenyl $2^{\prime}$ deoxyadenosine (116). ${ }^{42}$ Not altogether surprisingly, we obtained a $11 \%$ yield of cyclized product 117, which was in stark contrast to the $88 \%$ obtained with 118 in previous work (Scheme 25). ${ }^{41}$
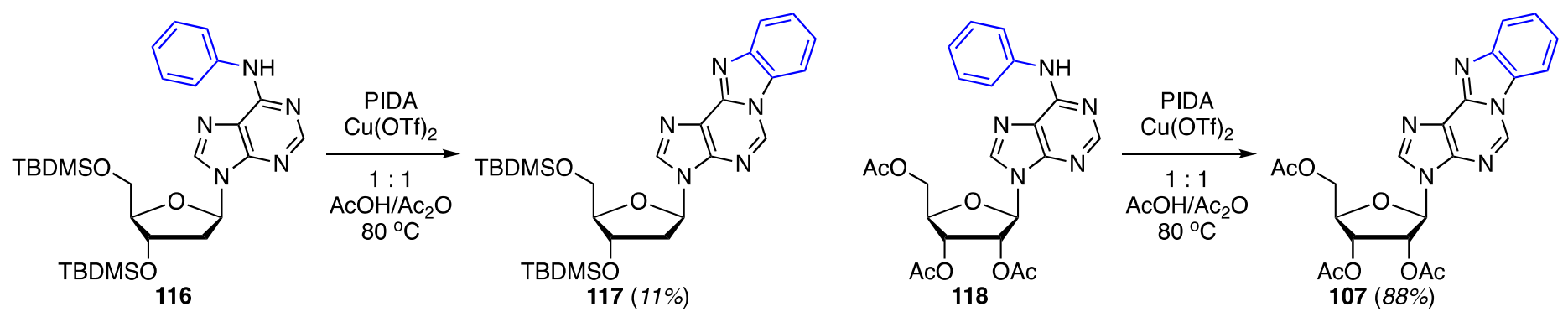

Scheme 25. Comparison of the reactivity of disilyl-protected $N^{6}$-phenyl 2'-deoxyadenosine to triacetylprotected $\mathrm{N}^{6}$-phenyl adenosine analogue.

This outcome prompted us to undertake a screening of conditions. We found that $\mathrm{Cu}(\mathrm{OTf})_{2}$ in $\mathrm{AcOH} / \mathrm{Ac}_{2} \mathrm{O}$, at $80{ }^{\circ} \mathrm{C}$, was clearly detrimental, and in switching the reaction solvent to $\mathrm{MeCN}$ the yield improved from 11 to $39 \%$, while in $\mathrm{MeCN}$ at $55{ }^{\circ} \mathrm{C}$ without $\mathrm{Cu}(\mathrm{OTf})_{2}$ a good $61 \%$ yield of 117 was realized. $\mathrm{PhMe}, \mathrm{ClCH}_{2} \mathrm{CH}_{2} \mathrm{CL}, \mathrm{DMF}$, 1,4-dioxane, and $\mathrm{MeNO}_{2}$ were investigated as solvents and yields ranged from 7-46\%. However, in $\mathrm{CF}_{3} \mathrm{CH}_{2} \mathrm{OH}$ 
(TFE) at $55{ }^{\circ} \mathrm{C}$, a good $75 \%$ yield of 117 was observed, and in $\left(\mathrm{CF}_{3}\right)_{2} \mathrm{CHOH}$ (HFIP), the reaction was faster in a comparable yield. With 1.3 equiv. of PIDA in HFIP at rt, an excellent $92 \%$ yield of $\mathbf{1 1 7}$ was obtained.

Using these conditions, several $t$-BuMe ${ }_{2}$ Si-protected $N^{6}$-aryl $2^{\prime}$-deoxyadenosine and adenosine analogues were assessed for this cyclization. In addition, one acetate-protected 2 '-deoxyadenosine derivative and one acetate-protected adenosine derivative were also evaluated. The products from these reactions are summarized in Figure 7.

From these data, it can be clearly seen that most product yields were in the $80-92 \%$ range. The only case where a low yield was obtained was in the case of the naphthyl derivative (122, 38\%). Notably, a low $40 \%$ yield was obtained with $\mathrm{Cu}(\mathrm{OTf})_{2} /$ PIDA (115 in Scheme 23). In fact the cyclization to 122 was conducted in $\mathrm{MeCN}$ as the substrate decomposed in HFIP. In our work, we isolated a second compound in this reaction and it was assigned imino quinone structure 123 on the basis of a ${ }^{13} \mathrm{C} \mathrm{NMR}$ resonance at $\delta=185.1 \mathrm{ppm}$ and the coupling constant of two coupled doublets in its ${ }^{1} \mathrm{H}$ NMR spectrum at $\delta=6.70$ and $7.05 \mathrm{ppm}(\mathrm{J} \approx 10.5 \mathrm{~Hz})$.
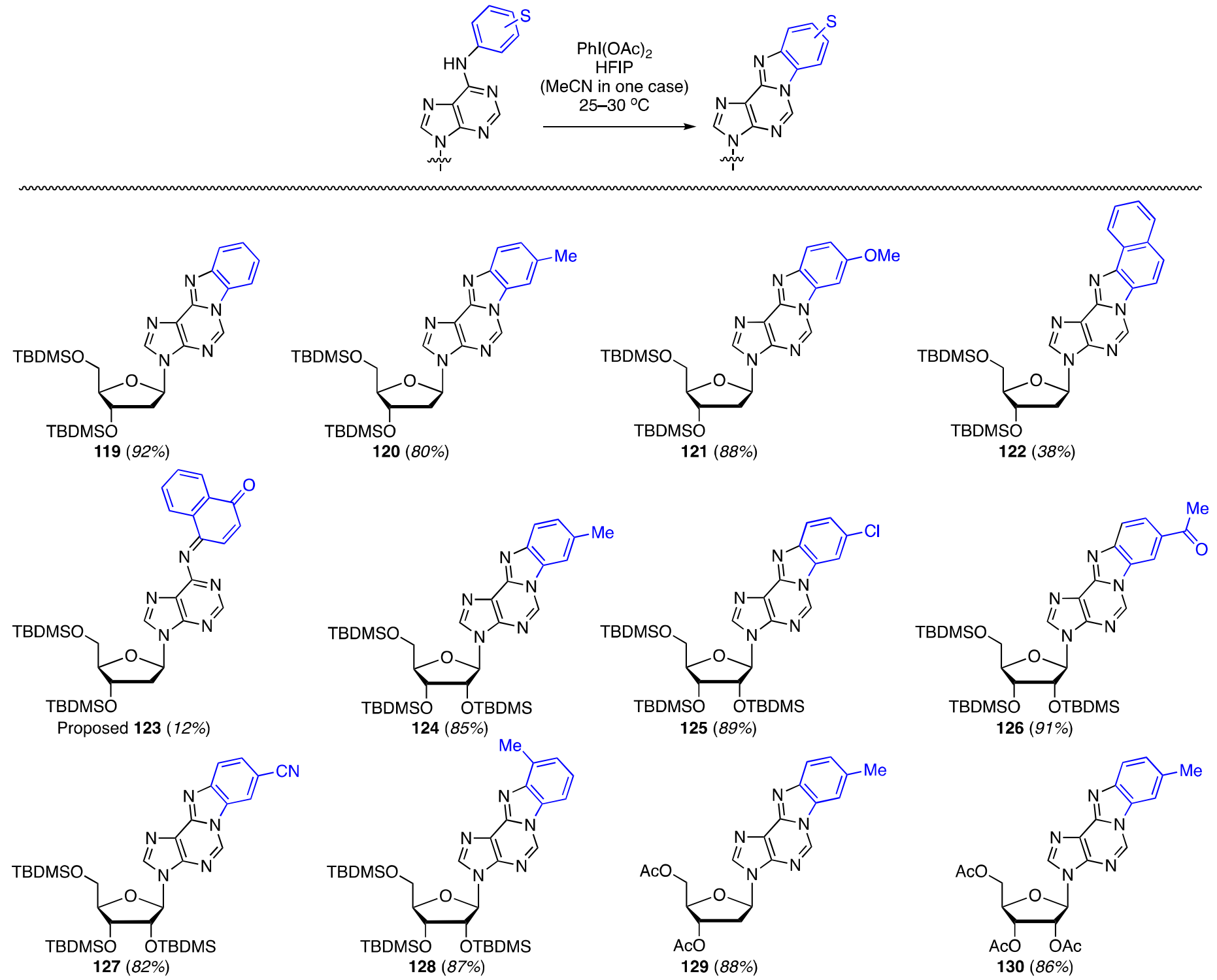

Scheme 26. Compounds synthesized using PIDA in HFIP, at room temperature. 
Notably this chemistry is applicable to silyl- and acetyl-protected 2'-deoxyribonucleosides, as well as to silyl- and acetyl-protected ribosyl analogues. Furthermore, this chemistry appears scalable, because both compounds, 119 and 126, could be prepared on $0.9 \mathrm{mmol}$ scale, in yields of 86 and 93\%, respectively.

The mechanism of this transformation was probed using butylated hydroxytoluene (BHT) and 1,1diphenylethene (DPE) as radical inhibitors. In three reactions, leading to 119, 121, and 127, either no product formation or only trace of product formation was observed, with substantial amounts of recovered starting material even with a single equivalent of the radical inhibitor. These data indicate that these reactions may proceed via radical cations or radicals (Scheme 26). Fluorinated solvents such as TFE and HFIP are known to stabilize radical cations ${ }^{43,44}$ that can be formed in reactions involving hypervalent iodine reagents.

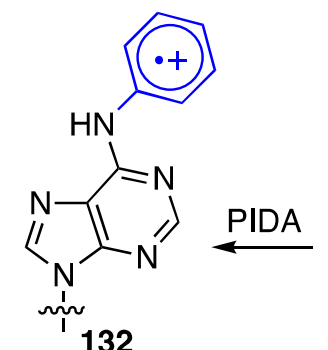

132

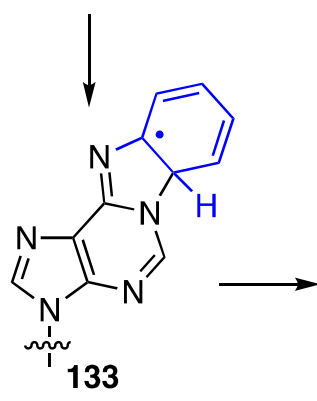

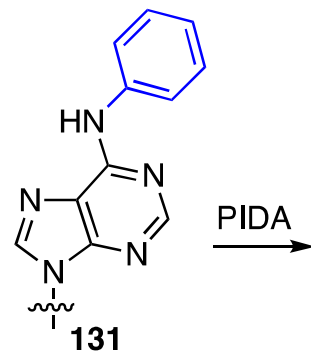

131

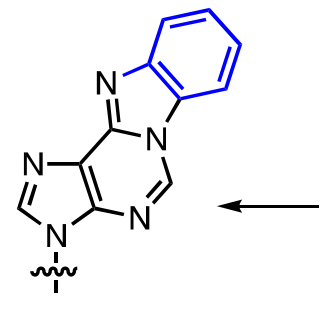

Product
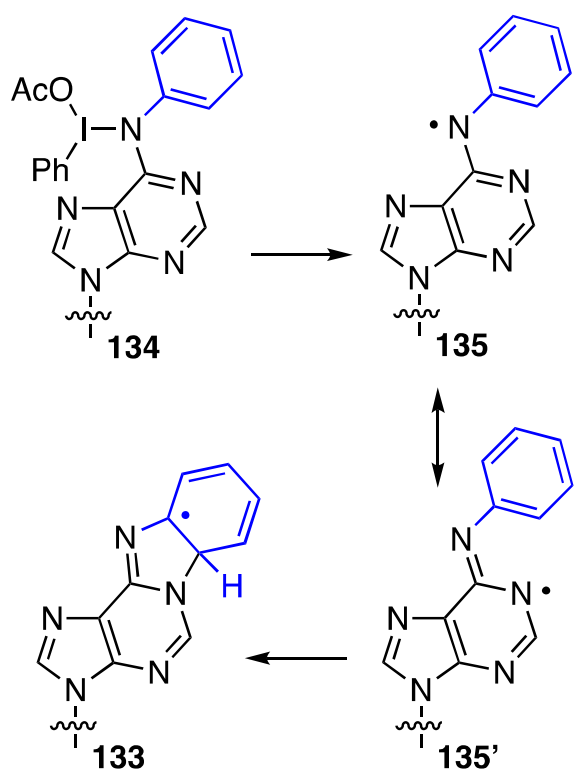

135

Scheme 27. A possible mechanistic pathway for the cyclization reaction with PIDA in HFIP.

A possible mechanism of this reaction could proceed via either formation of radical cation 132 that can cyclize to radical 133, or PIDA can react at the exocyclic amino group leading to 134 . Fragmentation of 134 by a radical pathway can result in a resonance stabilized, nitrogen-centered radical (135/135') that can also cyclize to 133. Loss of a hydrogen radical from 133 will then lead to product. Notably, Phl was isolated in the $0.9 \mathrm{mmol}-\mathrm{scale}$ reactions. Also, the products displayed interesting fluorescence properties.

\section{Pd-Catalyzed and Metal-Free Intramolecular $\mathbf{C}-\mathbf{N}$ Bond Formation Leading to Isomeric Carbazolyl and Benzimidazolyl Purine Nucleoside Analogues Using PIDA}

Most recently, we have studied cyclization of $\mathrm{N}^{6}$-biarylyl adenine nucleosides. ${ }^{46}$ With these compounds two cyclization modes are possible; with the exocyclic $\mathrm{N}^{6}$ atom leading to carbazolyl nucleoside analogues (route 1 ) or with the $\mathrm{N}^{1}$ ring nitrogen atom leading to aryl benzimidazolyl nucleoside derivatives (route 2; Scheme 28).

We first investigated Pd-catalyzed reactions of 11 substrates (136-146) shown in Figure 7. The reactions involving $\mathrm{Pd}(\mathrm{OAc})_{2}$ and PIDA are anticipated to proceed via a $\mathrm{Pd}^{\prime \prime} / \mathrm{Pd}^{\mathrm{IV}}$ mechanism, where the hypervalent iodine reagent acts as an oxidant for the metal. In PhMe, at $55^{\circ} \mathrm{C}$, reactions were observed with all substrates 
except the trifluoromethyl-substituted ones (145 and 146). The ribosyl precursors 137 and 140 reacted comparably to the respective deoxy analogues 136 and 139. Reaction of the $p$-cyano precursor 144 gave a low product yield and in this case an iminoquinone byproduct 144' was also isolated and characterized.
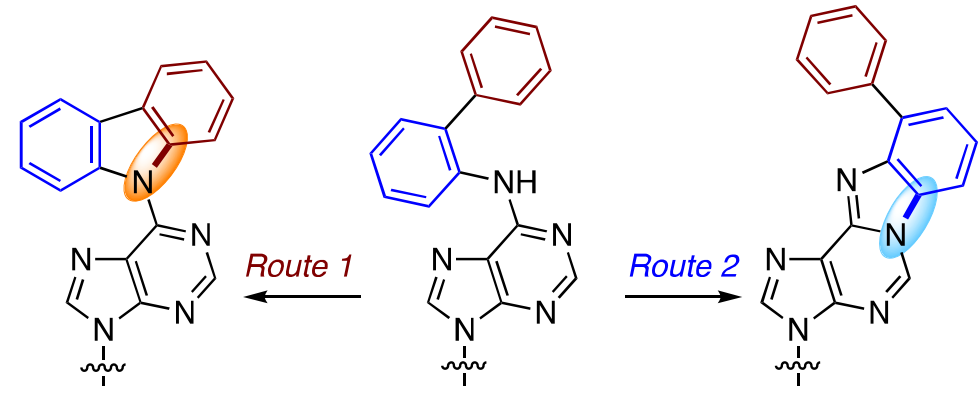

Scheme 28. Two possible cyclization modes with $\mathrm{N}^{6}$-biarylyl adenine nucleoside derivatives.

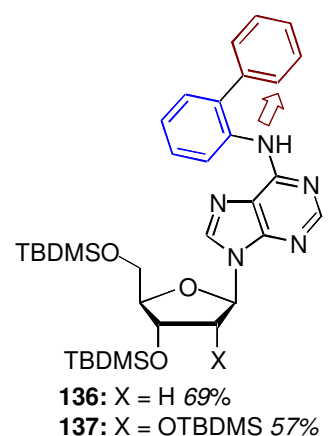

137: $X=$ OTBDMS $57 \%$

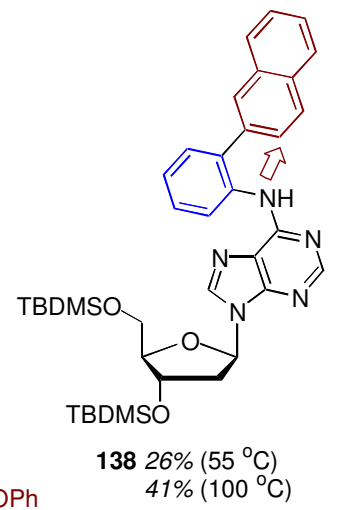

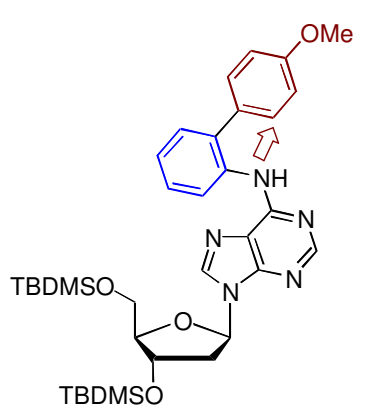

$14136 \%$

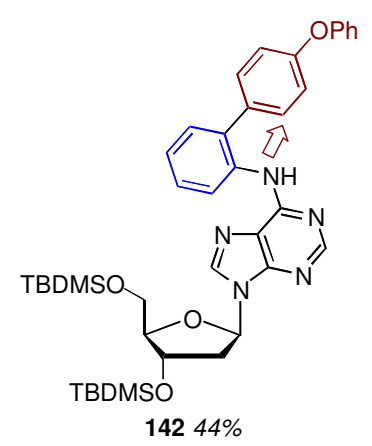

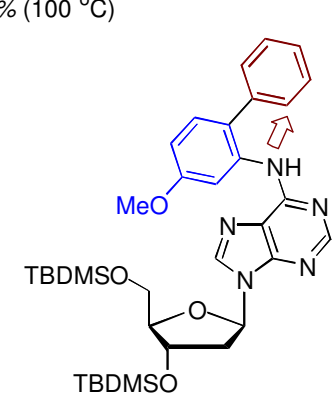

$14331 \%$
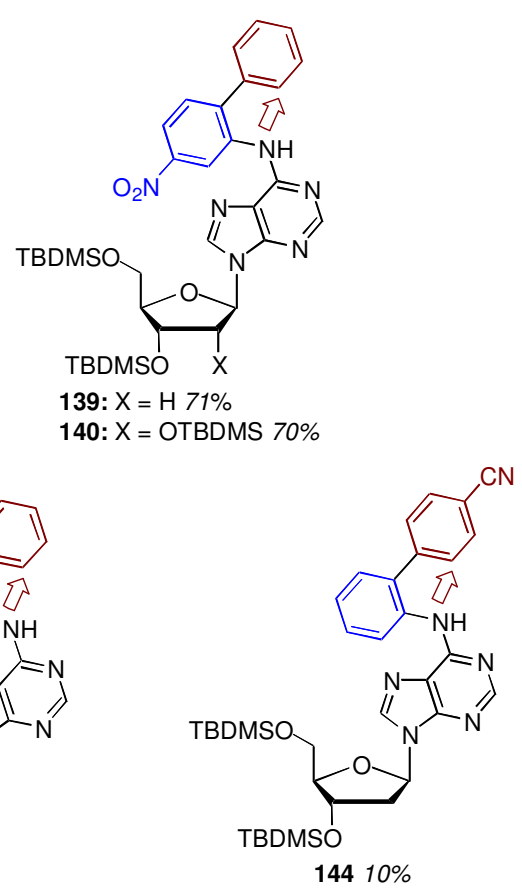
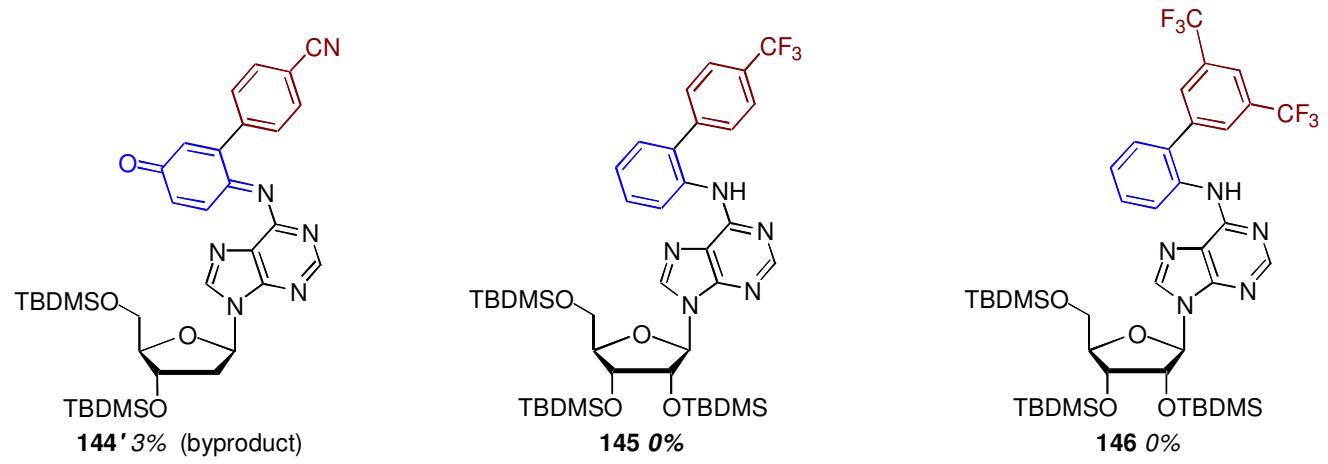

Figure 7. $\mathrm{N}^{6}$-biarylyl adenine nucleoside substrates used for the reactions involving $\mathrm{Pd}(\mathrm{OAc})_{2} / \mathrm{PIDA}$ (in cases where reaction was observed, red arrows indicate the site of $\mathrm{C}-\mathrm{N}$ bond formation and the ensuing carbazolyl product yields are shown). 


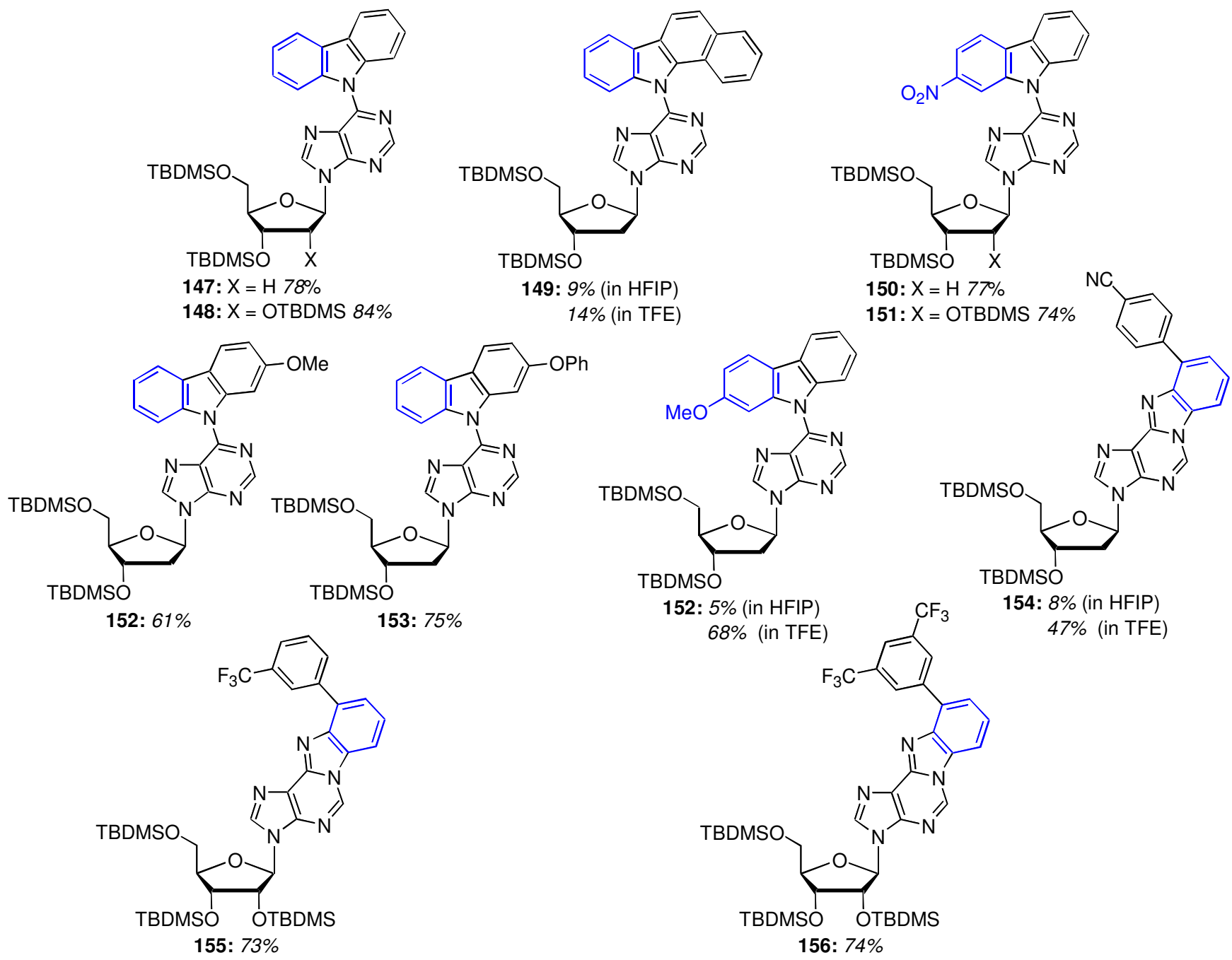

Figure 8. Products obtained in the fluorinated solvents in the absence of any catalyst.

In the course of investigating solvent effects for the Pd-catalyzed reaction, we found that cylization of compound 136 also occurred in HFIP and TFE as solvents, at $55{ }^{\circ} \mathrm{C}$, but in the absence of a metal catalyst. Further investigations were conducted into this chemistry and the products from cyclization with PIDA in fluorinated solvents are shown in Figure 8.

Generally, these reactions in fluorinated solvents are superior to those catalyzed by Pd. With substrate 138, cyclization to the 1-position of naphthalene was observed, which is different from the outcome in the Pdmediated approach where ring-closure occurred at the 2-position. Some substrates were sensitive to the more acidic HFIP and better to superior results were obtained in the less acidic TFE. With biarylyl substrates that bore an electron-withdrawing group on the ring more remote from purine $(144,145$, and 146$)$, cyclization occurred on the proximal ring, resulting in benzimidazolyl products (154-156).

When the solvent was changed from HFIP (or TFE) to MeCN, an interesting outcome was observed. In many cases where exclusive formation of carbazolyl nucleoside analogues was observed in the fluorinated solvent, competing formation of benzimidazolyl nucleoside derivatives was observed in MeCN. The results from the reactions in $\mathrm{MeCN}$, at $55{ }^{\circ} \mathrm{C}$, are summarized in Table 1 . The only exceptions to benzimidazole formation were methoxy-substituted substrates 141 and 143. However, when the methoxy group in 141 was replaced with a phenoxy moiety in 142, a small amount of the benzimidazole isomer was observed. Electron- 
withdrawing substituents on the ring remote from the purine led to benzimidazole formation in good yields (substrates 144-146).

Table 1. Results from the PIDA-mediated cyclization reactions of substrates 136-143, 145, and 146 in MeCN

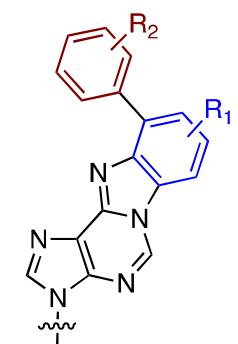

154-162
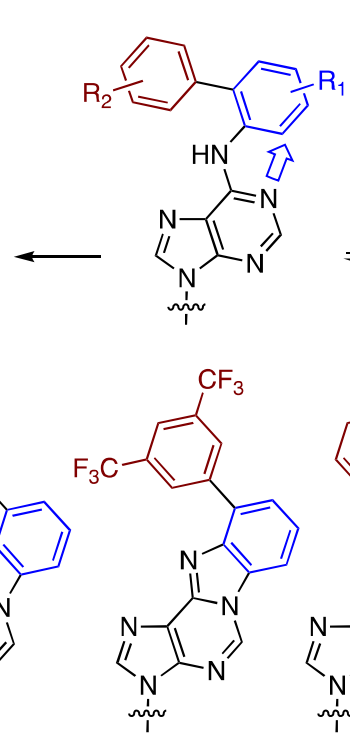

156

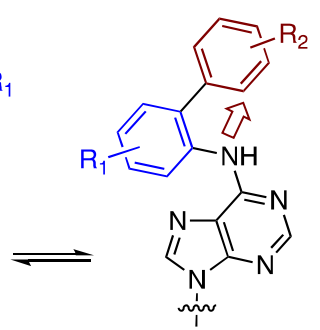

1

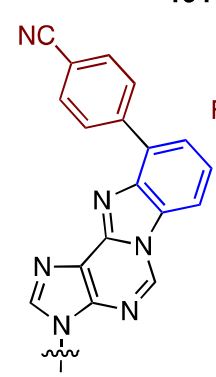

154

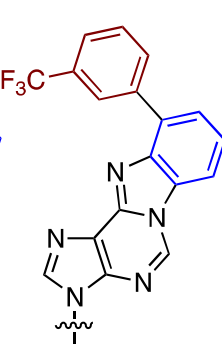

155

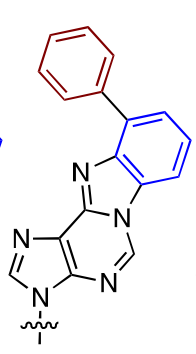

157 and 158

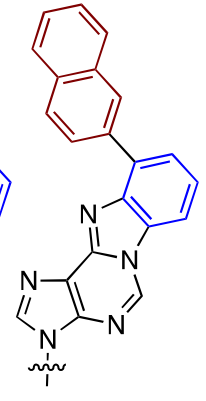

159

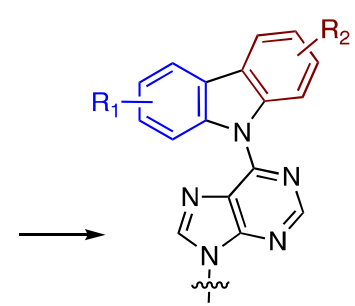

147-153

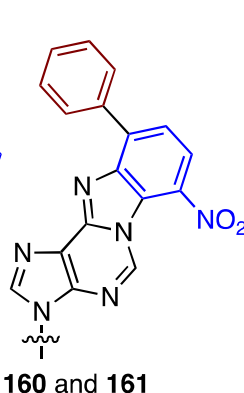

160 and 161

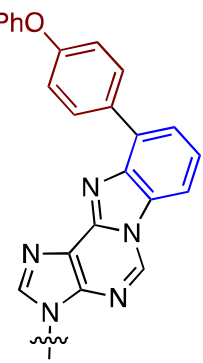

162

\begin{tabular}{|c|c|c|c|}
\hline Entry & Substrate & Carbazole & Benzimidazole \\
\hline 1 & 136 & $147: 15 \%$ & $157: 45 \%$ \\
\hline 2 & 137 & $148: 16 \%$ & $158: 58 \%$ \\
\hline 3 & 138 & $149: 50 \%$ & $159: 18 \%$ \\
\hline 4 & 139 & $150: 47 \%$ & $160: 30 \%$ \\
\hline 5 & 140 & $151: 35 \%$ & $161: 37 \%$ \\
\hline 6 & 141 & $152: 70 \%$ & - \\
\hline 7 & 142 & $153: 66 \%$ & $162: 7 \%$ \\
\hline 8 & 143 & $152: 53 \%$ & - \\
\hline 9 & 144 & - & $154: 64 \%$ \\
\hline 10 & 145 & - & $155: 72 \%$ \\
\hline 11 & 146 & - & $156: 69 \%$ \\
\hline
\end{tabular}

DFT analysis showed that electron density at the nitrogen atoms in the purine ring decreased in the order: exocyclic $N^{6}>N^{1}>N^{3}>N^{7}>N^{9}$. This indicated that both the exocylic $N^{6}$ and the ring $N^{1}$ atoms can function as good nucleophiles. Interestingly, the electron density on the exocyclic $\mathrm{N}^{6}$ atom increased with introduction of electron-withdrawing substituents.

Whereas the $\mathrm{Pd}$-catalyzed reactions are anticipated to proceed via $\mathrm{Pd}^{\prime \prime} / \mathrm{Pd}^{\mathrm{IV}}$ redox via $\mathrm{C}-\mathrm{Pd} \sigma$-bond formation, the uncatalyzed reactions could involve interactions of the hypervalent iodine reagent with the nitrogen atoms of the purine ring, and proceed via either radical cations ${ }^{47-49}$ and/or nitrogen-centered radicals, ${ }^{50,51}$ and/or nitrenium ions. ${ }^{52-63}$ Extensive investigations with radical traps indicated that a single mechanism could not be ascribed to the uncatalyzed reactions and that overlapping mechanisms could be responsible for the products formed. 


\section{Conclusions}

In this review, we have summarized some very interesting and unique reactions promoted by hypervalent iodine reagents. These range from Pummerer-type reactions that can be utilized for the development of sulfur and selenium analogues of natural nucleosides, as well as unnatural nucleosides. These reagents can also be used to prepare phenylsulfanyl and phenylselenyl pyrimidine nucleoside analogues, isoxazolesubstituted and isoxazoline-based nucleoside analogues. The purinyl ring nitrogen atom can be used to direct $\mathrm{C}-\mathrm{H}$ bond activation by palladium and oxidation, where PIDA plays a crucial role as oxidant. PIDA can also be used for metal-catalyzed and metal-free $\mathrm{C}-\mathrm{N}$, intramolecular bond forming reaction, leading to novel benzimidazopurine nucleoside analogues as well as carbazolyl nucleoside derivatives. As evidenced in this review, it is a reasonable expectation that hypervalent iodine reagents will continue to contribute significantly to the synthesis and modifications of nucleosides and nucleoside analogues.

\section{Acknowledgements}

Support by National Science Foundation Grants CHE-1265687 to MKL (for nucleoside modification chemistry described herein), and by CHE-1565754 to BZ, is gratefully acknowledged. Infrastructural support at The City College of New York has been provided by National Institutes of Health Grant G12 MD007603 from the National Institute of Minority Health and Health Disparities. We thank Dr. S. Satishkumar for a critical reading of this manuscript.

\section{References}

1. Modified Nucleosides: In Biochemistry, Biotechnology and Medicine; Herdewijn, P. Ed.; Wiley-VCH: Weinheim, 2008.

2. Nucleoside Triphosphates and Their Analogs: Chemistry, Biotechnology, and Biological Applications; Vaghefi, M. Ed.; CRC Press: Boca Raton, FL, 2005.

3. Antiviral Nucleosides: Chiral Synthesis and Chemotherapy; Chu, C. K. Ed.; Elsevier: Amsterdam, 2003.

4. Jordheim, L. P.; Durantel, D.; Zoulim, F.; Dumontet, C. Nature Rev. Drug Discov. 2013, 12, 447-464. https://doi.org/10.1038/nrd4010

5. Damaraju, V. L.; Damaraju, S.; Young, J. D.; Baldwin, S. A.; Mackey, J.; Sawyer M. B.; Cass, C. E. Oncogene 2003, 22, 7524-7536. https://doi.org/10.1038/sj.onc.1206952

6. Huryn, D. M.; Okabe, M. Chem. Rev. 1992, 92, 1745-1768. https://doi.org/10.1021/cr00016a004

7. Antiviral Drug Discovery for Emerging Diseases and Bioterrorism Threats; Torrance, P. F. Ed.; John Wiley.: Hoboken, NJ, 2005.

8. Niu, G.; Tan, H. Trends Microbiol. 2015, 23, 110-119.

https://doi.org/10.1016/i.tim.2014.10.007

9. Isono, K. J. Antibiot. 1988, 41, 1711-1739.

https://doi.org/10.7164/antibiotics.41.1711 
10. Knapp, S. Chem. Rev. 1995, 95, 1859-1876.

https://doi.org/10.1021/cr00038a006

11. Matarazzo A.; Hudson, R. H. E. Tetrahedron 2015, 71, 1627-1657.

https://doi.org/10.1016/i.tet.2014.12.066

12. Su, X.; Xiao, X.; Zhang C.; Zhao, M. Appl. Spectrosc. 2012, 66, 1249-1262.

https://doi.org/10.1366/12-06803

13. Tanpure, A. A.; Pawar M. G.; Srivatsan, S. G. Isr. J. Chem. 2013, 53, 366-378.

https://doi.org/10.1002/ijch.201300010

14. Sinkeldam, R. W.; Greco N. J.; Tor, Y. Chem. Rev. 2010, 110, 2579-2619.

https://doi.org/10.1021/cr900301e

15. Wilson J. N.; Kool, E. T. Org. Biomol. Chem. 2006, 4, 4265-4274.

https://doi.org/10.1039/b612284c

16. Wiebe, L. I. Braz. Arch. Biol. Technol. 2007, 50, 445-459.

https://doi.org/10.1590/S1516-89132007000300011

17. Hawkins, M. E. In Topics in Fluorescence Spectroscopy, Vol. 7: DNA Technology; Lakowicz, J. R. Ed.; Kluwer Academic/Plenum: New York, 2003; pp 151-175.

18. Molecular Imaging Probes for Cancer Research; Chen, X. Ed.; World Scientific Publishing: Singapore, 2012.

19. Current Directions in Radiopharmaceutical Research and Development; Mather, S. J. Ed.; Kluwer: Dordrecht, The Netherlands, 1996.

20. Nishizono, N.; Baba, R.; Nakamura, C.; Oda, K.; Machida, M. Org. Biomol. Chem. 2003, 1, 3692-3697. https://doi.org/10.1039/B305644A

21. Nishizono, N.; Soma, K.; Baba, R.; Machida, M.; Oda, K. Heterocycles 2008, 75, 619-634.

https://doi.org/10.3987/COM-07-11250

22. Jeong, L. S.; Jin, D. Z.; Kim, H. O.; Shin, D. H.; Moon, H. R.; Gunaga, P.; Chun, M. W.; Kim, Y.-C.; Melman, N.; Gao, Z.-G.; Jacobson, K. A. J. Med. Chem. 2003, 46, 3775-3777.

https://doi.org/10.1021/im034098e

23. Nishizono, N.; Akama, Y.; Agata, M.; Sugo, M.; Yamaguchi, Y.; Oda, K. Tetrahedron 2011, 67, 358-363. https://doi.org/10.1016/j.tet.2010.11.038

24. Taniike, H.; Inagaki, Y.; Matsuda, A.; Minakawa. N. Tetrahedron 2011, 67, 7977-7982. https://doi.org/10.1016/i.tet.2011.08.020

25. Ishii, K.; Saito-Tarashima, N.; Ota, M.; Yamamoto, S.; Okamoto, Y.; Tanaka, Y.; Minakawa, N. Tetrahedron 2016, 72, 6589-6594.

https://doi.org/10.1016/j.tet.2016.08.071

26. Yu, J.; Kim, J.-H.; Lee, H. W.; Alexander, V.; Ahn, H.-C.; Choi, W. J.; Choi, J.; Jeong, L. S. Chem.-Eur. J. 2013, 19, 5528-5532.

https://doi.org/10.1002/chem.201300741

27. Yoshimura, Y.; Ohta, M.; Imahori, T.; Imamichi, T.; Takahata, H. Org. Lett. 2008, 10, 3449-3452. https://doi.org/10.1021/ol8012155

28. Yoshimura, Y.; Kan-no, H.; Kiran, Y. B.; Natori, Y.; Saito, Y.; Takahata, H. Synthesis 2012, 44, 1163-1170. https://doi.org/10.1055/s-0031-1290749

29. Kan-no, H.; Saito, Y.; Omoto, S.; Minato, S.; Wakamatsu, H.; Natori, Y.; Imamichi, T.; Takahata, H.; Yoshimura, Y. Synthesis 2014, 46, 879-886.

https://doi.org/10.1055/s-0033-1340663 
30. Roh, K. R.; Chang, H. K.; Kim, Y. H. Heterocycles 1998, 48, 437-441.

https://doi.org/10.3987/COM-97-8074

31. Cheng, D. P.; Chen, Z. C.; Zheng, Q. G. J. Chem. Res. (S) 2002, 624-625.

32. Jawalekar, A. M.; Reubsaet, E.; Rutjes, F. P. J. T.; van Delft, F. L. Chem. Commun. 2011, 47, 3198-3200. https://doi.org/10.1039/c0cc04646a

33. Balalas, T.; Peperidou, C.; Hadjipavlou-Litina, D. J.; Litinas, K. E. Synthesis 2016, 48, 281-292.

34. Guo, H.-M.; Jiang, L.-L.; Niu, H.-Y.; Rao, W.-H.; Liang, L.; Mao, R.-Z.; Li, D.-Y.; Qu, G.-R. Org. Lett. 2011, 13, 2008-2011.

https://doi.org/10.1021/ol200405w

35. Lakshman, M. K.; Deb, A. C.; Chamala, R. R.; Pradhan, P.; Pratap, R. Angew. Chem., Int. Ed. 2011, 50, 11400-11404.

https://doi.org/10.1002/anie.201104035

36. Guo, H.-M.; Rao, W.-H.; Niu, H.-Y.; Jiang, L.-L.; Meng, G.; Jin, J.-J.; Yang, X.-N.; Qu, G.-R. Chem. Commun. 2011, 47, 5608-5610.

https://doi.org/10.1039/c1cc11135c

37. Chamala, R. R.; Parrish, D.; Pradhan, P.; Lakshman, M. K. J. Org. Chem. 2013, 78, 7423-7435. https://doi.org/10.1021/j04008282

38. Rios, A. C.; Yu, H. T.; Tor, Y. J. Phys. Org. Chem. 2015, 28, 173-180.

https://doi.org/10.1002/poc.3318

39. Bercaw, J. E.; Durrell, A. C.; Gray, H. B.; Green, J. C.; Hazari, N.; Labinger, J. A.; Winkler, J. R. Inorg. Chem. 2010, 49, 1801-1810.

https://doi.org/10.1021/ic902189g

40. Powers, D. C.; Xiao, D. Y.; Geibel, M. A. L.; Ritter, T. J. Am. Chem. Soc. 2010, 132, 14530-14536. https://doi.org/10.1021/ja1054274

41. Powers, D. C.; Geibel, M. A. L.; Klein, J. E. M. N.; Ritter, T. J. Am. Chem. Soc. 2009, 131, 17050-17051. https://doi.org/10.1021/ja906935c

42. Qu, G.-R.; Liang, L.; Niu, H.-Y.; Rao, W.-H.; Guo, H.-M.; Fossey, J. S. Org. Lett. 2012, 14, 4494-4497. https://doi.org/10.1021/ol301848v

43. Satishkumar, S.; Lakshman, M. K. Chem. Commun. 2017, 53, 2226-2229. https://doi.org/10.1039/C6CC07722F

44. Eberson, L.; Hartshorn, M. P.; Persson, O.; Radner, F. Chem. Commun. 1996, 2105-2112. https://doi.org/10.1039/cc9960002105

45. Eberson, L.; Hartshorn, M. P.; Persson, O. J. Chem. Soc., Perkin Trans. 2 1995, 1735-1744. https://doi.org/10.1039/p29950001735

46. Satishkumar, S.; Poudapally, S.; Vuram, P. K.; Gurram, V.; Pottabathini, N.; Sebastian, D.; Yang, L.; Pradhan, P.; Lakshman, M. K. ChemCatChem. 2017, 9, 4050-4069.

https://doi.org/10.1002/cctc.201700918

47. Kita, Y.; Dohi, T. Chem. Rec. 2015, 15, 886-906.

https://doi.org/10.1002/tcr.201500020

48. Kita, Y.; Takada, T.; Mihara, S.; Whelan, B. A.; Tohma, H. J. Org. Chem. 1995, 60, 7144-7148. https://doi.org/10.1021/jo00127a018

49. Kita, Y.; Tohma, H.; Hatanaka, K.; Takada, T.; Fujita, S.; Mitoh, S.; Sakurai, H.; Oka, S. J. Am. Chem. Soc. 1994, 116, 3684-3691.

https://doi.org/10.1021/ja00088a003 
50. Togo, H.; Harada, Y.; Yokoyama, M. J. Org. Chem. 2000, 65, 926-929. https://doi.org/10.1021/jo991419e

51. Togo, H.; Hoshina, Y.; Muraki, T.; Nakayama, H.; Yokoyama, M. J. Org. Chem. 1998, 63, 5193-5200. https://doi.org/10.1021/jo980450y

52. Antonchick, A. P.; Samanta, R.; Kulikov, K.; Lategahn, J. Angew. Chem. Int. Ed. 2011, 50, 8605-8608. https://doi.org/10.1002/anie.201102984

53. Samanta, R.; Bauer, J. O.; Strohmann,C.; Antonchick, A. P. Org. Lett. 2012, 14, 5518-5521. https://doi.org/10.1021/ol302607y

54. Liu, H.; Wang, X.; Gu, Y. Org. Biomol. Chem. 2011, 9, 1614-1620.

https://doi.org/10.1039/c0ob00749h

55. Tellitu, I.; Domínguez, E. Trends Heterocycl. Chem. 2011, 15, 23-32.

56. Amano, Y.; Nishiyama, S. Tetrahedron Lett. 2006, 47, 6505-6507. https://doi.org/10.1016/j.tetlet.2006.07.050

57. Wardrop, D. J.; Burge, M. S. J. Org. Chem. 2005, 70, 10271-10284. https://doi.org/10.1021/jo051252r

58. Misu, Y.; Togo, H. Org. Biomol. Chem. 2003, 1, 1342-1346. https://doi.org/10.1039/b301330h

59. Wardrop, D. J.; Zhang, W.; Landrie, C. L. Tetrahedron Lett. 2004, 45, 4229-4231. https://doi.org/10.1016/i.tetlet.2004.04.028

60. Wardrop, D. J.; Burge, M. S. Chem. Commun. 2004, 1230-1231. https://doi.org/10.1039/B403081H

61. Kikugawa, Y.; Nagashima, A.; Sakamoto, T.; Miyazawa, E.; Shiiya, M. J. Org. Chem. 2003, 68, 6739-6744. https://doi.org/10.1021/jo0347009

62. Itoh, N.; Sakamoto, T.; Miyazawa, E.; Kikugawa, Y. J. Org. Chem. 2002, 67, 7424-7428. https://doi.org/10.1021/jo0260847

63. Kikugawa, Y.; Kawase, M. Chem. Lett. 1990, 581-582. https://doi.org/10.1246/cl.1990.581

\section{Authors' Biographies}

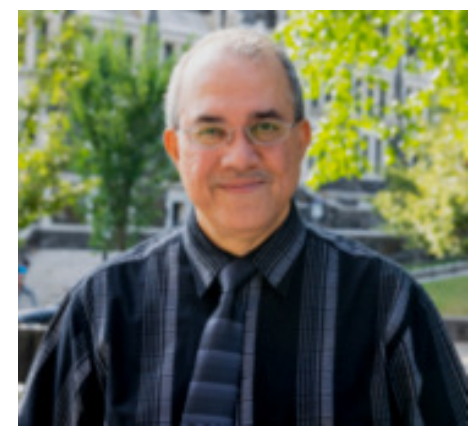

Mahesh K. Lakshman obtained the B.Sc. and M.Sc. degrees from the University of Bombay (Mumbai, India) and the Ph.D. degree from the University of Oklahoma. After completing a postdoctoral appointment at the National Institutes of Health (NIDDK), under a Fogarty Fellowship, he entered the private sector. He then joined the University of North Dakota and later moved to The City College of New York (The City University of New York system), where he rose to the level of Professor. He has served as Executive Officer of The City 
University of New York Ph.D. Program in Chemistry and as one of two Vice Chairs of the Department of Chemistry \& Biochemistry at The City College of New York. His research has been supported by the National Science Foundation and the National Institutes of Health. He is a Fellow of the Royal Society of Chemistry, a recipient of a Japan Society for the Promotion of Science Fellowship, and has been a Visiting Professor at the Indian Institute of Technology Bombay. In 2015, he and Professor Fumi Nagatsugi (Tohoku University) GuestEdited a special issue of Molecules, entitled Nucleoside Modifications.

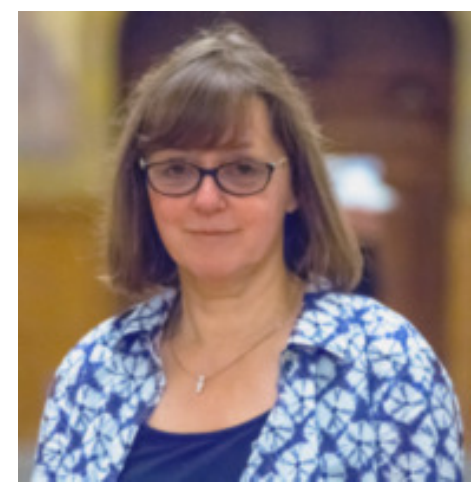

Barbara Zajc obtained the B.Sc., M.Sc., and Ph.D. degrees from the University of Ljubljana (Slovenia). She then undertook a sabbatical at the National Institutes of Health (NIDDK), under a Fogarty Fellowship. She returned to the University of Ljubljana where she was Docent and was then promoted to Associate Professor. In 2001 she moved to The City College of New York (The City University of New York system) as Associate Professor where she is now at the level of Professor. Her research has been supported by the National Science Foundation and the National Institutes of Health. 